\title{
A wall-wake model for the turbulence structure of boundary layers. Part 1. Extension of the attached eddy hypothesis
}

\author{
By A. E. PERRY AND IVAN MARUŠIĆ \\ Department of Mechanical and Manufacturing Engineering, University of Melbourne, Parkville, \\ Victoria 3052, Australia
}

(Received 14 January 1994 and in revised form 22 April 1995)

The attached eddy hypothesis developed for zero pressure gradient boundary layers and for pipe flow is extended here to boundary layers with arbitrary streamwise pressure gradients, both favourable and adverse. It is found that in order to obtain the correct quantitative results for all components of the Reynolds stresses, two basic types of eddy structure geometries are required. The first type, called type-A, is interpreted to give a 'wall structure' and the second, referred to as type- $B$, gives a 'wake structure'. This is in analogy with the conventional mean velocity formulation of Coles where the velocity is decomposed into a law of the wall and a law of the wake.

If the above mean velocity formulation is accepted, then in principle, once the eddy geometries are fixed for the two eddy types, all Reynolds stresses and associated spectra contributed from the attached eddies can be computed without any further empirical constants. This is done by using the momentum equation and certain convolution integrals developed here based on the attached eddy hypothesis. The theory is developed using data from equilibrium and quasi-equilibrium flows. In Part 2 the authors' non-equilibrium data are used.

\section{Introduction}

This paper describes extensions to the work on the attached eddy hypothesis of Townsend (1976) and the model based on this developed at Melbourne by Perry and various co-workers. In this paper all developments in the attached eddy hypothesis over the last decade are brought together and expressed with a consistent notation and set of normalizing conditions. It is then extended to boundary layers with imposed streamwise pressure gradients. Past work has been mainly concerned with pipe flow and zero pressure gradient flow.

In most past work the eddies in the attached eddy hypothesis were used only notionally to illustrate functional forms and trends and to aid in dimensional arguments. No serious attempt to produce quantitative results has been made. To do this would require a knowledge of precise eddy shapes. In this paper some tentative shapes are tried and quantitative comparisons are made with data. Although precise shapes for representative eddies are not known and probably will never be known, the authors are convinced that definite conclusions can be drawn concerning important gross properties of the attached eddy shapes. 
By the term 'attached eddies' we mean a set of geometrically similar eddies consisting of a range of length scales with individual length scales proportional to the distance at which the eddy is located from, or extends above, the wall. The essential finding here is that there are two types of attached eddies which are responsible for most of the turbulent kinetic energy and Reynolds shear stresses. These will be referred to as type-A and type-B eddies. In all previous work only type-A eddies were used in the modelling.

For type-A eddies, the vortex lines extend to the wall while for type-B the vortex lines undulate in the spanwise direction but do not reach the boundary. It is tentatively proposed here that the type-A eddies are responsible for a universal wall structure and for the mean-flow logarithmic law of the wall, which here extends throughout the layer, whereas type-B structures produce a wake structure (which contributes to 'non-universal' turbulence intensities in the wall region) and are responsible for the mean flow wake component. This is consistent with the ideas expressed by Coles $(1956,1957)$ who was concerned only with mean velocities. The wall structure is identical to the 'pure wall' flow which occurs in equilibrium sink flow where the Coles wake factor is zero. From this hopefully a unified theory can be constructed, valid for favourable, zero and adverse pressure gradient flows.

As in Perry, Henbest \& Chong (1986) it is shown from spectral considerations that there must be further structures which contribute to the high-wavenumber motions. These will be collectively referred to as type-C eddies. These are probably detached eddies, i.e. their length scale is not related directly to the distance they are located from the wall. The eddies in the Kolmogorov inertial subrange and dissipation range form a subset of type- $C$ structures. These make only a minor contribution to the Reynolds normal stresses and are considered in more detail in Part 2 (Marušic \& Perry 1995).

Perry, Marušić \& Li (1994) recently illustrated one way the attached eddy hypothesis might be incorporated into a 'closure scheme' for computing the evolution of the mean flow parameters of a turbulent boundary layer developing in a pressure gradient. Only type-A eddies were used in the formulation and by making certain hypotheses about the weighting functions $T$ and $w$ to be defined later, a connection between the mean velocity defect and the Reynolds shear stress was formulated. Unfortunately if all stress components are to be accurately described by the attached eddy model it is found here that it is almost certain that more than one type of eddy structure is necessary. Perhaps in future work this extended model might be useful in formulating a 'closure scheme' along the lines of Perry et al. (1994). We make no attempt to do this here.

\section{Shear stress distributions}

Analytical expressions for the shear stress distribution are used in development of the model. The analysis in this section was developed by Perry et al. (1994) but further details are highlighted here and in Appendix A for the purpose of the present paper.

A slightly generalized form of the Coles (1956) logarithmic wall of the wall and law of the wake formulation is

$$
\frac{U}{U_{\tau}}=\frac{1}{\kappa} \log \left[\frac{z U_{\tau}}{v}\right]+A+\frac{\Pi}{\kappa} W_{c}[\eta, \Pi]
$$


and in the velocity defect form

$$
\begin{aligned}
\frac{U_{1}-U}{U_{\tau}} & =f[\eta, \Pi] \\
& =-\frac{1}{\kappa} \log \eta+\frac{\Pi}{\kappa} W_{c}[1, \Pi]-\frac{\Pi}{\kappa} W_{c}[\eta, \Pi] .
\end{aligned}
$$

Here $U_{1}$ is the local free-stream velocity, $U$ is the local mean velocity, $U_{\tau}$ is the shear velocity, $v$ is the fluid kinematic viscosity, $W_{c}$ is the Coles wake function, $\kappa$ is the Kármán constant $(=0.41), A$ is the universal law of the wall constant $(=5.1), z$ is the distance normal to the wall and $\eta=z / \delta_{c}$ where $\delta_{c}$ is the boundary layer thickness.

It is assumed that the mean flow is two-dimensional and that streamwise derivatives of normal stresses can be neglected. The mean continuity equation is

$$
\frac{\partial U}{\partial x}+\frac{\partial W}{\partial z}=0
$$

where $W$ is the mean velocity component normal to the wall and the streamwise momentum equation is

$$
U \frac{\partial U}{\partial x}+W \frac{\partial U}{\partial z}=-\frac{1}{\rho} \frac{\mathrm{d} P}{\mathrm{~d} x}+\frac{1}{\rho} \frac{\partial \tau}{\partial z}
$$

where $P$ is the free-stream static pressure and $\tau$ is the local effective shear stress given by

$$
\frac{\tau}{\rho}=-\overline{u_{1} u_{3}}+v \frac{\partial U}{\partial z} .
$$

Here $v \partial U / \partial z$ is the viscous contribution and $-\overline{u_{1} u_{3}}$ is the Reynolds (kinematic) shear stress where $u_{1}$ and $u_{3}$ are the fluctuating components of velocity in the $x$ - and $z$-directions respectively and the overbar denotes a time average. For $z U_{\tau} / v>50$ the viscous contribution in (5) is negligible. It should be emphasized here that the authors are assuming that Reynolds numbers are sufficiently large that the viscous zone can be completely neglected and that the logarithmic defect law goes right to the wall. For momentum balance purposes this is found to be accurate even for moderate Reynolds numbers (see Perry, et al. 1994).

It can be shown from all the above equations (see Appendix A) that

$$
\frac{\tau}{\tau_{0}}=f_{1}[\eta, \Pi, S]+f_{2}[\eta, \Pi, S] \delta_{c} \frac{\mathrm{d} \Pi}{\mathrm{d} x}+f_{3}[\eta, \Pi, S] \frac{\delta_{c}}{U_{1}} \frac{\mathrm{d} U_{1}}{\mathrm{~d} x}
$$

where

$$
S=\frac{U_{1}}{U_{\tau}}=\left(2 / C_{f}^{\prime}\right)^{1 / 2},
$$

where $C_{f}^{\prime}$ is the local skin friction coefficient, given by

$$
C_{f}^{\prime}=\frac{\tau_{0}}{\frac{1}{2} \rho U_{1}^{2}}
$$

where $\tau_{0}$ is the wall shear stress. The functions $f_{1}$ and $f_{2}$ and $f_{3}$ are somewhat complicated and are given in Appendix A. The Lewkowicz (1982) formulation for $W_{c}[\eta, \Pi]$ will be introduced into the equations. This formulation ensures that $\partial U / \partial z=0$ at $\eta=1$, and is given by

$$
W_{c}[\eta, \Pi]=2 \eta^{2}(3-2 \eta)-\frac{1}{\Pi} \eta^{2}(1-\eta)(1-2 \eta) .
$$


With (9), $f_{1}, f_{2}$ and $f_{3}$ have the general form

$$
f_{i}[\eta, \Pi, S]=\frac{N_{i}[\eta, \Pi, S]}{D_{i}[\eta, \Pi, S]}
$$

where

$$
N_{i}[\eta, \Pi, S]=\sum_{q=0, r=0}^{\gamma, \delta} A_{q, \Gamma} \eta^{q}(\log \eta)^{r} \sum_{m=0, n=0}^{\alpha, \beta} B_{m, n} \Pi^{m} S^{n}
$$

and

$$
D_{i}[\eta, \Pi, S]=\sum_{m=0, n=0}^{\theta, \varphi} C_{m, n} \Pi^{m} S^{n} .
$$

In all cases $\gamma=9, \delta=2, \alpha=4, \beta=3, \theta=2, \psi=2$ and terms involving logarithms are restricted to $\eta \log \eta, \eta^{3} \log \eta, \eta^{4} \log \eta, \eta^{5} \log \eta$ and $\eta(\log \eta)^{2}$. There are no $\eta^{2}$ terms anywhere and not all $A_{q, r}, B_{m, n}$ and $C_{m, n}$ are finite. The fully expanded forms of $f_{1}, f_{2}$ and $f_{3}$ can be generated quite easily using Mathematica or any equivalent package, and are given in an internal report by Perry and Marušic (1993).

For finite $\eta$ and $\Pi$, the $f_{i}$ can be written as

$$
\left.\begin{array}{rl}
f_{1}[\eta, \Pi, S] & =\frac{a_{1} S^{2}+a_{2} S+a_{3}}{b_{1} S^{2}+b_{2} S+b_{3}}, \\
f_{2}[\eta, \Pi, S] & =\frac{c_{1} S^{3}+c_{2} S^{2}+c_{3} S+c_{4}}{e_{1} S^{2}+e_{2} S+e_{3}}, \\
f_{3}[\eta, \Pi, S] & =\frac{q_{1} S^{3}+q_{2} S^{2}+q_{3} S+q_{4}}{h_{1} S^{2}+h_{2} S+h_{3}},
\end{array}\right\}
$$

where $a_{1}, a_{2}$ etc. are polynomials in $\eta$ and $\Pi$.

From equation (6) it can be seen that there are three components of shear stress as seen schematically in figure 1 for a typical adverse pressure gradient layer. The first term gives a distribution which resembles that of a zero pressure gradient layer. The second term gives a negative contribution if $\delta_{c} \mathrm{~d} \Pi / \mathrm{d} x$ is positive and the third term gives a positive contribution for a negative $\left(\delta_{c} / U_{1}\right)\left(\mathrm{d} U_{1} / \mathrm{d} x\right)$. For the case of a favourable pressure gradient $\delta_{c} \mathrm{~d} \Pi / \mathrm{d} x$ is usually negative and $\left(\delta_{c} / U_{1}\right)\left(\mathrm{d} U_{1} / \mathrm{d} x\right)$ is positive and so the second and third components of the shear stress reverse sign.

It is usual to express the pressure gradient parameter in terms of the Clauser (1954, 1956) parameter $\beta$, thus

$$
\frac{\delta_{c}}{U_{1}} \frac{\mathrm{d} U_{1}}{\mathrm{~d} x}=-\frac{\beta}{C_{1} S}
$$

where $\beta$ as defined by Clauser is

$$
\beta=\frac{\delta^{*}}{\tau_{0}} \frac{\mathrm{d} p}{\mathrm{~d} x},
$$

where $\delta^{*}$ is the displacement thickness and $C_{1}$ is given by

$$
C_{1}[\Pi]=\int_{0}^{1} f[\eta, \Pi] \mathrm{d} \eta
$$

Equation (6) can now be written as

$$
\frac{\tau}{\tau_{0}}=f_{1}[\eta, \Pi, S]+g_{1}[\eta, \Pi, S] \zeta+g_{2}[\eta, \Pi, S] \beta
$$



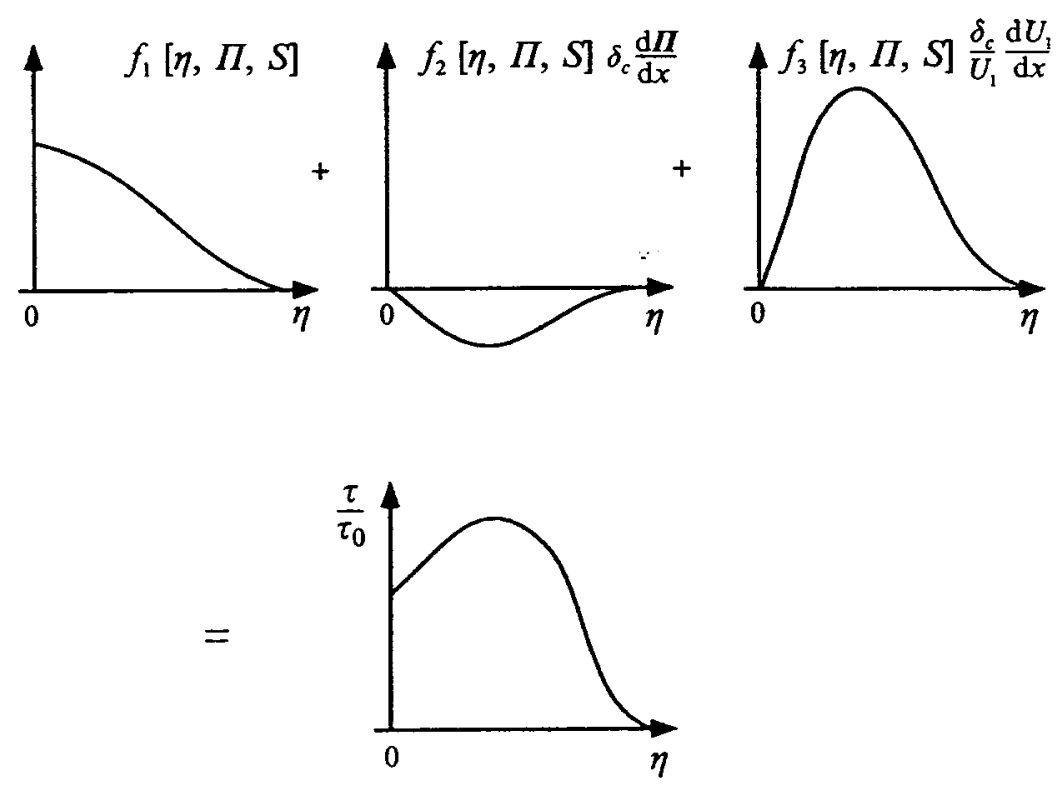

FIGURE 1. Components of equation (6) for a typical adverse pressure gradient case. After Perry et al. (1994).

where

$$
g_{1}=\frac{f_{2}}{S}, \quad g_{2}=-\frac{f_{3}}{C_{1} S}, \quad \zeta=S \delta_{c} \frac{\mathrm{d} \Pi}{\mathrm{d} x} .
$$

From the structure of the equations shown in (11) it can be seen that for a finite $\zeta$ and $\beta, \tau / \tau_{0}$ remains finite and becomes independent of $S$ as $S \rightarrow \infty$.

\subsection{Equilibrium layers}

For equilibrium layers as originally defined by Clauser, $\mathrm{d} \Pi / \mathrm{d} x=0$ and this gives self-similar mean velocity defect profiles. It can be seen that for a fixed $\beta$ as $S \rightarrow \infty$ we also have self-similar shear stress profiles. This is a more acceptable definition of equilibrium layers, i.e. we have self-similarity in both velocity defect and shear stress profiles. This is the definition used by Rotta (1962) and Townsend (1961) but here it is approached asymptotically for $S$ sufficiently large which would require unrealistically high Reynolds numbers. Rotta (1962) and others have shown that for finite $S$, this type of smooth-wall equilibrium layer can occur only in sink flow. Perry $(1968,1992)$ and Perry et al. (1994) have shown that approximate equilibrium flow would occur at finite $S$ if $\beta=\beta[S, \Pi]$ is allowed to vary appropriately with $S$ before reaching its asymptotic value $\beta_{a}[\Pi]=\beta[\infty, \Pi]$ which is a function only of $\Pi$. This essentially means that it may be possible to produce a class of layers where the shear stress distributions is fixed once $\Pi$ is fixed. Furthermore, a wider class of layers might exist where the above is still true but $\Pi$ is permitted to vary slowly with $x$. This class is referred to as 'quasi-equilibrium' layers by Perry et al. (1994) and it is obvious that for these layers to exist

$$
\alpha[\eta, \Pi, S, \zeta, \beta]=\frac{g_{1}[\eta, \Pi, S] \zeta}{\tau / \tau_{0}} \ll 1
$$




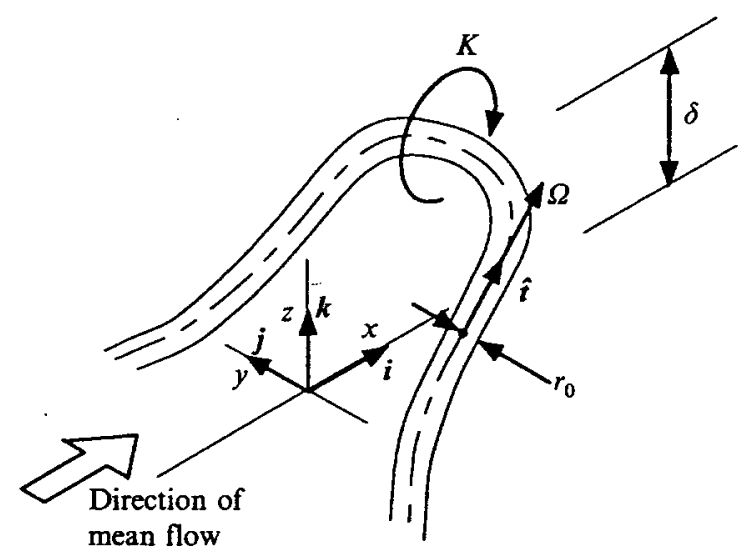

FIGURE 2. Sketch of a representative attached eddy.

As a measure of how close a layer is to quasi-equilibrium we could evaluate and tabulate a quantity $\sigma$ defined thus:

$$
\sigma=\alpha[0.5, \Pi, S, \zeta, \beta]
$$

An interesting point is that for equilibrium and quasi-equilibrium layers the hypotheses of Perry et al. (1994) state that the mean non-dimensional defect distributions and the shear stress distributions are fixed by the parameter $\Pi$ alone. This means we have automatically, whether one believes in a gradient diffusion mechanism or not, a universal distribution of eddy viscosity $\epsilon$ given by $\epsilon /\left(\delta_{c} U_{\tau}\right)=\psi[\eta, \Pi]$, where $\psi$ is a universal function. This was also proposed by Clauser (1956). However one should keep in mind that this can only be approximately true and once condition (16) is violated, as is often the case (e.g. in Part 2), this eddy viscosity formulation breaks down.

Another interesting point here is that according to equation (10) a linear stress layer' does not generally occur (analytically at least) in a developing boundary layer as was often assumed in the past. One exception to this is in the logarithmic wall region of a zero pressure gradient layer with $S \rightarrow \infty$ (see Perry, Li \& Marušic 1991 and Perry et al. 1994).

\section{Formulation of the attached eddy hypothesis}

Figure 2 shows schematically a representative attached eddy in a turbulent boundary layer. It leans in the streamwise direction with a fixed orientation as it slips relative to the boundary. This velocity of slip is assumed to occur across a thin viscous region which could be regarded as a vortex sheet. The eddy height is $\delta$. The vortex tube shown is symmetrical about the $(x, z)$-plane and for $z / \delta$ sufficiently large the plane of the loop is probably at about $45^{\circ}$ to the wall. It should be mentioned that this representative eddy should be regarded as a statistical concept which possesses the gross features of an assemblage of eddies. An instantaneous realizable eddy will probably be a gross distortion of this representative eddy.

It will be assumed that a turbulent boundary layer is made up of a random array of such eddies distributed over the surface with an average density proportional to $1 / \delta^{2}$. In previous work, for example Perry \& Chong (1982), Perry et al. (1986), and Perry, Lim \& Henbest (1987), this was referred to as a 'hierarchy' because it really 
consists of eddies at different stages of stretching and hence of different heights and shapes. Here we are replacing such eddies with a random array of 'representative eddies' which are all of the same height, shape and velocity scale. Furthermore, it is assumed that a range of geometrically similar arrays of eddies exists with the scale $\delta$ ranging from $\delta_{1}$, the smallest eddy $\left(\delta_{1}=O\left(100 v / U_{\tau}\right)\right)$ to $\delta_{c}$, the boundary layer thickness. Since we are integrating over a range of scales, individual departures of actual eddies from the representative eddies will be washed out. In the case of rough walls it has been suggested that $\delta_{1}$ is related to the roughness scale (Perry et al. 1987 and Perry \& $\mathrm{Li} 1990$ ). Let $K$ be the circulation of the vortex loop. Let $r_{0}$ be the effective radius of the vortex 'rod' forming the loop, and let $\Omega_{0}$ be the effective vorticity within the rod such that

$$
K=\pi r_{0}^{2} \Omega_{0}
$$

Let the characteristic velocity scale $U_{0}$ of the eddy be defined such that

$$
U_{0} \delta=\frac{K}{2 \pi}
$$

and therefore

$$
\frac{U_{0}}{\delta}=\frac{K}{2 \pi \delta^{2}}=\frac{\pi r_{0}^{2} \Omega_{0}}{2 \pi \delta^{2}}=\frac{1}{2} q^{2} \Omega_{0},
$$

where $q=r_{0} / \delta$ which is a geometric constant. Let the average streamwise and spanwise spacing of the eddies be $k_{x} \delta$ and $k_{y} \delta$ respectively, where $k_{x}$ and $k_{y}$ are geometric constants. The mean spanwise vorticity $\mathrm{d} U / \mathrm{d} z$ for one scale of eddies (or for one hierarchy) is given by

$$
\xi_{H}[z / \delta]=\frac{\Omega_{0}}{k_{x} k_{y}} \int_{-\infty}^{\infty} \int_{-\infty}^{\infty} \frac{\Omega}{\Omega_{0}}(\hat{t} \cdot j) \mathrm{d}(x / \delta) \mathrm{d}(y / \delta),
$$

that is

$$
\xi_{H}[z / \delta]=\frac{1}{k_{x} k_{y}} \frac{U_{0}}{\delta} \frac{2}{q^{2}} \int_{-\infty}^{\infty} \int_{-\infty}^{\infty} \frac{\Omega}{\Omega_{0}}(\hat{\boldsymbol{t}} \cdot j) \mathrm{d}(x / \delta) \mathrm{d}(y / \delta) .
$$

Let $P_{H}[\delta]$ be the probability density function for eddy scales $\delta$. Hence the mean vorticity for a range of scales from $\delta_{1}$ to $\delta_{c}$ is

$$
\frac{\mathrm{d} U}{\mathrm{~d} z}=\int_{\delta_{1}}^{\delta_{c}} \xi_{H}[z / \delta] P_{H}[\delta] \mathrm{d} \delta .
$$

Let

$$
P_{H}[\delta]=\frac{1}{\delta} D_{1}\left[\delta / \delta_{c}\right]
$$

where $D_{1}$ is a weighting function. If $D_{1}$ is a constant then $P_{H}[\delta]$ gives a -1 power law p.d.f. which under certain conditions leads to the well-known logarithmic velocity defect distribution. However, in general $D_{1}\left[\delta / \delta_{c}\right]$ will vary with $\delta / \delta_{c}$ and will be a measure of the departure of $P_{H}[\delta]$ from a -1 power law p.d.f.

From all of the above it is not difficult to show that

$$
\frac{\mathrm{d} U / U_{\tau}}{\mathrm{d} z}=\int_{\delta_{1}}^{\delta_{c}} f[z / \delta] Q\left[\delta / \delta_{c}\right] D\left[\delta / \delta_{c}\right] \frac{1}{\delta^{2}} \mathrm{~d} \delta
$$

where

$$
f[z / \delta]=\frac{2}{q^{2}} \int_{-\infty}^{\infty} \int_{-\infty}^{\infty} \frac{\Omega}{\Omega_{0}}(\hat{\boldsymbol{t}} \cdot j) \mathrm{d}(x / \delta) \mathrm{d}(y / \delta),
$$


and is a function only of the geometry of the non-dimensional vorticity distribution. Also

$$
Q\left[\delta / \delta_{c}\right]=\frac{U_{0}}{U_{\tau}}
$$

and is a measure of how the characteristic velocity scale to friction velocity ratio varies with eddy scale, while

$$
D\left[\delta / \delta_{c}\right]=\frac{1}{k_{x} k_{y}} D_{1}\left[\delta / \delta_{c}\right]
$$

and this is a measure of how the p.d.f. of eddy scales departs from a -1 power law and how the eddy population density on the $(x, y, 0)$ surface varies with eddy scale.

Equation (25) can be transformed using the following logarithmic variables. Let

$$
\lambda=\log [\delta / z], \quad \lambda_{E}=\log \left[\delta_{c} / z\right], \quad \lambda_{1}=\log \left[\delta_{1} / z\right]
$$

and let $U_{D}^{*}=\left(U_{1}-U\right) / U_{\tau}$. This leads to the following convolution integral:

$$
\frac{\mathrm{d} U_{D}^{*}}{\mathrm{~d} \lambda_{E}}=\int_{-\infty}^{\infty} h[\lambda] \mathrm{e}^{-\lambda} T\left[\lambda-\lambda_{E}\right] w\left[\lambda-\lambda_{E}\right] \mathrm{d} \lambda,
$$

where

$$
w\left[\hat{\lambda}-\lambda_{E}\right]=D\left[\delta / \delta_{c}\right], \quad T\left[\lambda-\lambda_{E}\right]=Q\left[\delta / \delta_{c}\right], \quad h[\lambda]=f[z / \delta] .
$$

The functions $w$ and $T$ switch to zero when $\lambda<\lambda_{1}$ and $\lambda>\lambda_{E}$. This effectively controls the limits of the integration.

The streamwise, spanwise and normal-to-the-wall velocity components are denoted by the index values of 1,2 and 3 respectively.

The Reynolds stresses may be computed using the Biot-Savart law applied to an isolated eddy together with its image in the wall. Let the contribution to the Reynolds stress from one scale of eddies be denoted by $\Delta\left(\overline{u_{i} \bar{u}_{j}}\right)$ and is given by

$$
\Delta\left(\overline{u_{i} u_{j}}\right)=\frac{U_{0}^{2}}{k_{x} k_{y}} \int_{-\infty}^{\infty} \int_{-\infty}^{\infty} \frac{V_{i} V_{j}}{U_{0}^{2}} \mathrm{~d}(x / \delta) \mathrm{d}(y / \delta),
$$

where $V_{i}$ are velocity components computed from the Biot-Savart law. (A discussion of how this relates to mean and fluctuating velocity components is given in Appendix B.) Therefore

$$
\overline{u_{i} u_{j}}=\int_{\delta_{1}}^{\delta_{c}} \Delta\left(\overline{u_{i} u_{j}}\right) P_{H}[\delta] \mathrm{d} \delta
$$

and then finally,

$$
\frac{\overline{u_{i} u_{j}}}{U_{\tau}^{2}}=\int_{\delta_{1}}^{\delta_{c}} I_{i j}[z / \delta] Q^{2}\left[\delta / \delta_{c}\right] D\left[\delta / \delta_{c}\right] \frac{1}{\delta} \mathrm{d} \delta,
$$

where $Q$ and $D$ are as before and

$$
I_{i j}\left[\frac{z}{\delta}\right]=\int_{-\infty}^{\infty} \int_{-\infty}^{\infty} \frac{V_{i} V_{j}}{U_{0}^{2}} \mathrm{~d}(x / \delta) \mathrm{d}(y / \delta) .
$$

It can be seen that the $I_{i j}[z / \delta]$ (the eddy intensity functions) are functions only of the geometry of the non-dimensional distribution of vorticity. Again using logarithmic variables we have

$$
\frac{\overline{u_{i} u_{j}}}{U_{\tau}^{2}}=\int_{-\infty}^{\infty} J_{i j}[\lambda] T^{2}\left[\lambda-\lambda_{E}\right] w\left[\lambda-\lambda_{E}\right] \mathrm{d} \lambda
$$


where

$$
J_{i j}[\lambda]=I_{i j}[z / \delta]
$$

Equation (30) which gives the mean velocity defect distribution and (36) which gives the Reynolds shear stress distribution are decoupled since the former depends on $T w$ and the latter on $T^{2} w$. Unless further hypotheses about $T$ and $w$ are introduced (as in Perry et al. 1994) we have no connection between (30) and (36) which will lead to closure for computing the streamwise evolution of the boundary layer.

Turbulence spectra can be computed in a similar way by a slight extension of the work of Perry et al. (1986). Here, their analysis will be repeated in terms of the notation used above. Using the Biot-Savart law, velocity signatures can be computed from an isolated eddy and its image, in terms of $x$ along lines of constant $y$ and $z$, i.e. $V_{i}[x]$ say. The Fourier transform in terms of non-dimensional quantities is given by

$$
F_{i}\left[k_{1} \delta, y / \delta, z / \delta\right]=\int_{-\infty}^{\infty} \frac{V_{i}}{U_{0}} \mathrm{e}^{-\mathrm{i} \not x k_{1} \delta(x / \delta)} \mathrm{d}(x / \delta)
$$

where $k_{1}$ is the streamwise wavenumber. The power spectral density of a random array of such eddies of scale $\delta$ is

$$
P_{i j}\left[k_{1} \delta, z / \delta\right]=U_{0}^{2} / k_{x} k_{y} \int_{-\infty}^{\infty} \operatorname{Re}\left\{F_{i}^{*} F_{j}\right\} \mathrm{d}(y / \delta)
$$

Here Re means 'the real part of' and the asterisk indicates a complex conjugate. Let us now express the power spectral density as a function of $k_{1} z$. Using the convention that the wavenumber argument indicates the non-dimensional wavenumber over which the spectral density is measured, then

$$
\phi_{i j}\left[k_{1} z, z / \delta\right]=\delta / z P_{i j}\left[k_{1} \delta, z / \delta\right]=\frac{U_{0}^{2}}{k_{x} k_{y}} G_{i j}\left[k_{1} z, z / \delta\right]
$$

where

$$
G_{i j}\left[k_{1} z, z / \delta\right]=\frac{\delta}{z} \int_{-\infty}^{\infty} \operatorname{Re}\left\{F_{i}^{*} F_{j}\right\} \mathrm{d}(y / \delta),
$$

and can be obtained from an isolated eddy and its image and is a function only of the eddy geometry.

As for earlier quantities, the spectral density for a range of scales is

$$
\Phi_{i j}\left[k_{1} z, z / \delta_{c}, z / \delta_{1}\right]=\int_{\delta_{1}}^{\delta_{c}} \phi_{i j}\left[k_{1} z, z / \delta\right] P_{H}[\delta] \mathrm{d} \delta
$$

and therefore

$$
\frac{\Phi_{i j}\left[k_{1} z, z / \delta_{c}, z / \delta_{1}\right]}{U_{\tau}^{2}} \cdot \int_{\delta_{1}}^{\delta_{c}} G_{i j}\left[k_{1} z, z / \delta\right] Q^{2}\left[\delta / \delta_{c}\right] D\left[\delta / \delta_{c}\right] \frac{1}{\delta} \mathrm{d} \delta .
$$

Multiplying both sides by $k_{1} z$ and using premultiplied spectra together with logarithmic variables we have

$$
\frac{\Psi_{i j}\left[\alpha_{z}, \lambda_{E}, \lambda_{1}\right]}{U_{\tau}^{2}}=\int_{-\infty}^{\infty} g_{i j}\left[\alpha_{z}, \lambda\right] T^{2}\left[\lambda-\lambda_{E}\right] w\left[\lambda-\lambda_{E}\right] \mathrm{d} \lambda,
$$

where

$$
\begin{gathered}
\Psi_{i j}\left[\alpha_{z}, \lambda_{E}, \lambda_{1}\right]=k_{1} z \Phi_{i j}\left[k_{1} z, z / \delta_{c}, z / \delta_{1}\right], \\
g_{i j}\left[\alpha_{z}, \hat{\lambda}\right]=k_{1} z G_{i j}\left[k_{1} z, z / \delta\right]
\end{gathered}
$$

and $\alpha_{z}=\log \left[k_{1} z\right]$ and $g_{i j}$ is a function only of eddy geometry. 

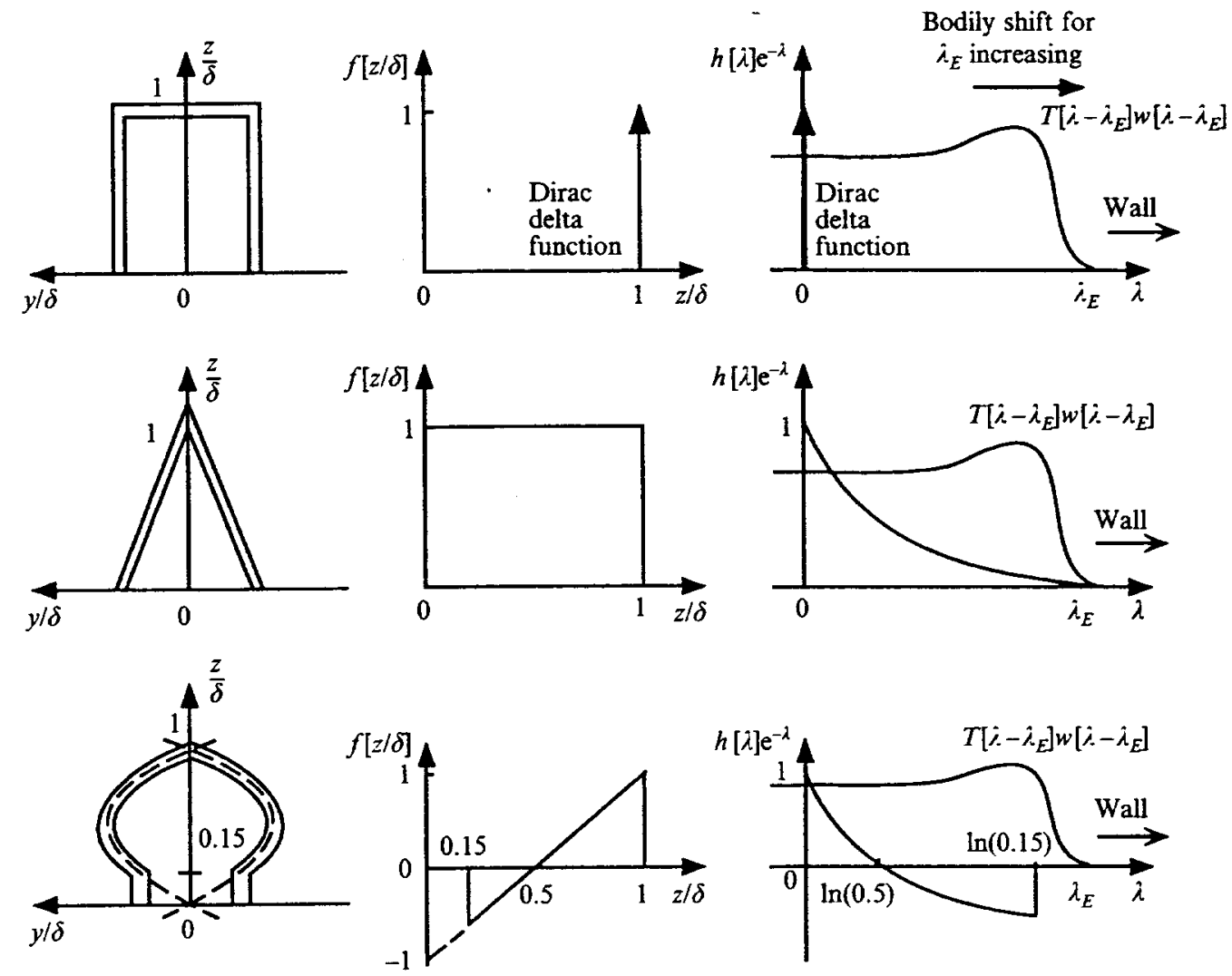

FIGURE 3. Different type-A attached eddies with their corresponding vorticity distributions and $T w$ weighting functions.

Finally, it can be shown that the eddy intensity function $J$ is related to the eddy spectral function $g$ by

$$
J_{i j}[\lambda]=\int_{-\infty}^{\infty} g_{i j}\left[\alpha_{z}, \lambda\right] \mathrm{d} \alpha_{z} .
$$

Actually it turns out to be quicker to compute eddy intensity functions using (44) rather than using (35). It is found that both methods give the same answer.

\section{Mean velocity and stress distributions in the fully turbulent wall region as} given by the attached eddy hypothesis

Eddy shapes of the type shown in figure 3 where the legs extend all the way to the wall will be called type-A eddies. Other eddy types will be considered later. Figure 3 shows a variety of type-A eddy shapes which lie on a plane inclined at $45^{\circ}$ to the wall in the streamwise direction together with the vorticity intensity functions $f[z / \delta]$ and $h[\lambda] \mathrm{e}^{-\lambda}$. Also shown is $T\left[\lambda-\lambda_{E}\right] w\left[\lambda-\lambda_{E}\right]$ superimposed on the $h[\lambda] \mathrm{e}^{-i}$ versus $\lambda$ plot. The integral of the product of $h\left[\lambda_{]}\right] \mathrm{e}^{-i}$ with $T\left[\lambda-\lambda_{E}\right] w\left[\lambda-\lambda_{E}\right]$ with $\lambda_{E} \rightarrow \infty$ gives the functional form for $\mathrm{d} U_{D}^{*} / \mathrm{d} \lambda_{E}$ close to the wall.

For $\lambda-\lambda_{E}$ large and negative (i.e. low $\delta / \delta_{c}$ ) $T$ and $w$ are taken to be constants, meaning a constant characteristic velocity scale and geometrically similar hierarchies 
(the eddies and their non-dimensional spacing are geometrically similar) and a -1 power law p.d.f. of eddy (hierarchy) scale.

It can be seen from figure 3 that for $\hat{\lambda}_{E}$ sufficiently large no matter what eddy shape is chosen, the integral of the product becomes a constant and from equation (30) $\mathrm{d} U_{D}^{*} / \mathrm{d} \lambda_{E}=$ constant, i.e.

$$
\frac{\mathrm{d}\left(U_{1}-U\right) / U_{\tau}}{\mathrm{d} \log \left[\delta_{c} / z\right]}=\frac{1}{\kappa}
$$

where $\kappa$ happens to be the Kármán constant, which is used in part to evaluate $T$ and $w$.

Figure 4 shows sketches of the eddy intensity functions $I_{13}, I_{11}, I_{22}$ and $I_{33}$ for typeA eddies. The important near-wall behaviour was first deduced by Townsend (1976) using inviscid boundary conditions. $I_{13}$ must be linear with $z / \delta$ as $z / \delta \rightarrow 0, I_{11}$ and $I_{22}$ must approach a constant and $I_{33}$ must be parabolic in $z / \delta$. These important features are shown for the corresponding $J_{i j}[\lambda]$ plotted versus $\lambda$ in the figure. Again, $T$ and $w$ are constant for sufficiently large and negative $\lambda-\lambda_{E}$. It can be seen from the figures that the convolution integral (36) leads to the following near-wall conditions:

$$
\text { for } \lambda_{E} \rightarrow \infty \quad-\frac{\overline{u_{1} u_{3}}}{U_{\tau}^{2}}=\text { const. }=1 \text {. }
$$

This constant must be unity (for sufficiently high Reynolds number) and this condition in part helps to evaluate $T$ and $w$. Next

$$
\text { for } \lambda_{E} \rightarrow \infty \quad \frac{\overline{u_{1}^{2}}}{U_{\tau}^{2}}=H_{1}+K_{1} g \hat{\lambda}_{E},
$$

where the constant $H_{1}$ in part depends on the form of $T^{2}\left[\lambda-\lambda_{E}\right] w\left[\lambda-\lambda_{E}\right]$ over the range $b$ shown in the figure. This in turn depends on outer flow conditions (such as the Coles wake factor), thus $H_{1}$ is a characteristic constant, but $K_{1} g$ is universal (= $A_{1}$ say) once an eddy shape has been fixed. A similar analysis applies for $\overline{u_{2}^{2}} / U_{\tau}^{2}$ and so

$$
\text { for } \lambda_{E} \rightarrow \infty \quad \frac{\overline{u_{1}^{2}}}{U_{\tau}^{2}}=H_{1}-A_{1} \log \left[z / \delta_{c}\right], \quad \frac{\overline{u_{2}^{2}}}{U_{\tau}^{2}}=H_{2}-A_{2} \log \left[z / \delta_{c}\right]
$$

For $\overline{u_{3}^{2}} / U_{\tau}^{2}$ it can be seen that

$$
\text { for } \lambda_{E} \rightarrow \infty \quad \frac{\overline{u_{3}^{2}}}{U_{\tau}^{2}}=K_{3}
$$

which is a universal constant. These forms were first arrived at by Townsend (1976).

Departure from these above relationships for increasing $z / \delta_{c}$ is controlled by how $T^{2}\left[\lambda-\lambda_{E}\right] w\left[\lambda-\lambda_{E}\right]$ behaves over the region $b$ shown in the figure. For example, the Reynolds shear stress gradients and higher derivatives with $z / \delta_{c}$ are controlled by this region $b$.

It will be noted that $\lambda_{1}$ is considered to be so small compared to $\lambda_{E}$, that it has not been shown on the diagram in figure 4 . However in figure 5 it has been included to illustrate the effect of the smallest attached eddy-scale cut-off and to illustrate an important Reynolds number effect. One can see that once $\lambda_{1}$ exceeds zero, i.e. $\delta_{1} / z$ becomes larger than 1 or $z<\delta_{1}$, then we are in the smallest attached eddy scale and the Reynolds shear stress drops rapidly to zero as $z / \delta_{c} \rightarrow 0$ and the total shear stress ratio $\tau / \tau_{0}$ must be taken up by viscous contributions as given in equation (5). To do 

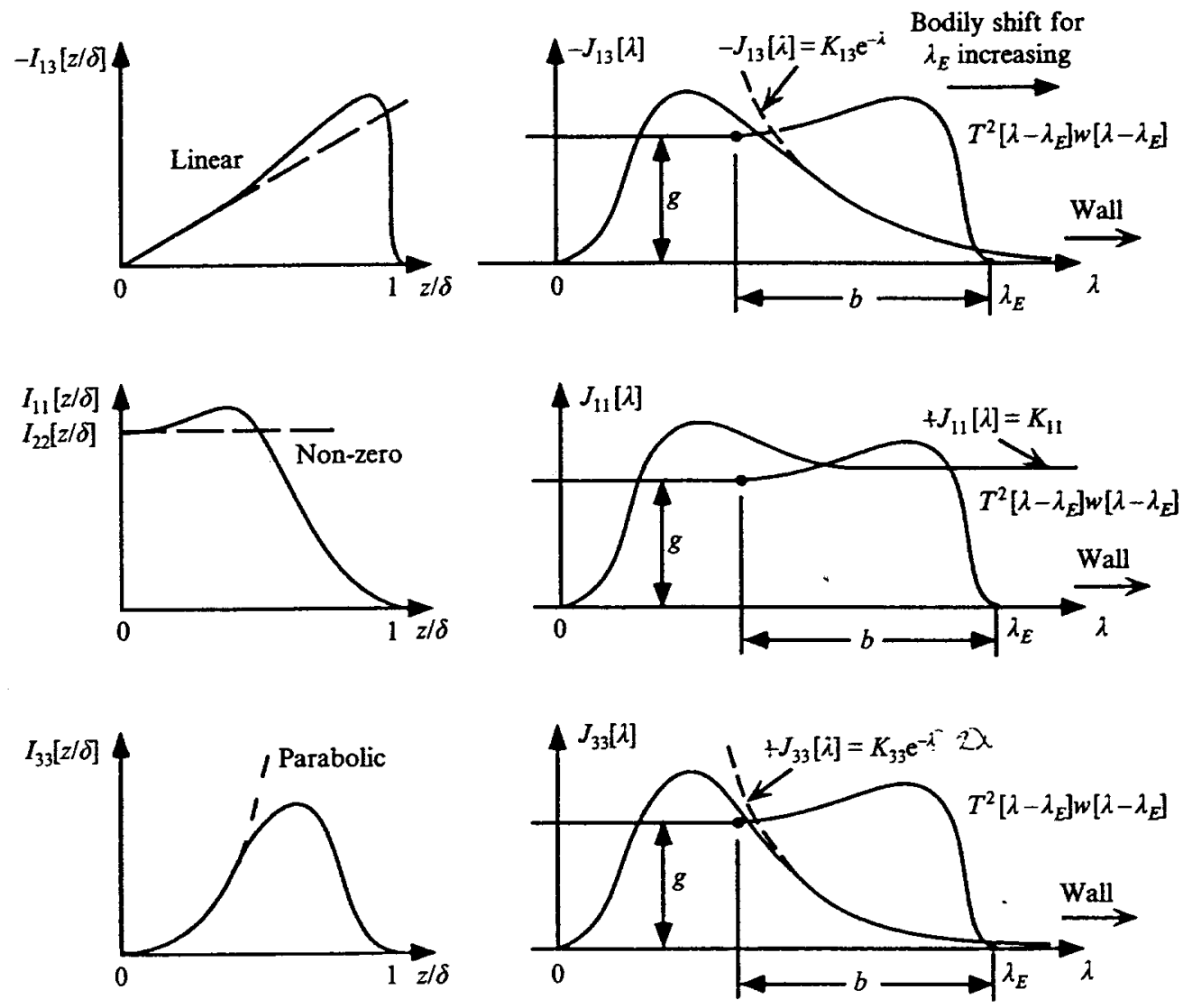

FIGURE 4. Typical eddy intensity functions for type-A eddies with $T^{2} w$ weighting functions.

this we must insert a viscous layer and buffer zone into the attached eddy model. In figure 5 it is seen that $\lambda_{E}-\lambda_{1}$ is an important parameter. Given $\lambda_{1} \approx 100 v / U_{\tau}$ then $\lambda_{E}-\lambda_{1}=\log \left(\delta_{c} U_{\tau} / 100 v\right)$ or $\log \left[K_{\tau} / 100\right]$ where $K_{\tau}$ is the Kármán number $\delta_{c} U_{\tau} / v$. The higher $K_{\tau}$ is, the larger is $\lambda_{E}-\lambda_{1}$ and hence the higher $\lambda_{E}$ can go before we experience this viscous cut-off. Hence the larger $K_{\tau}$ is, the smaller $z / \delta_{c}$ can become before reaching the viscous zone.

In all this work we are assuming that we are dealing with layers where $K_{\tau}$ is sufficiently large to allow $z / \delta_{c}$ to always be in the turbulent wall region, no matter how small we make it.

\section{Testing hypotheses with experiment using type-A eddies}

With the mathematical framework in place certain hypotheses concerning eddy shape can be made. The first hypothesis to be tested is that all eddies are of a universal shape and that the Reynolds stress distributions and spectra are controlled by variations of velocity scale and population density with eddy scale $\delta$. These are expressed by the function $T^{2}\left[i-\lambda_{E}\right] w\left[\lambda-\lambda_{E}\right]$.

It should be pointed out at the outset that the theoretical predictions are valid only beyond the viscous buffer zone and that all experimental measurements were made beyond this buffer zone. The condition $z / \delta_{c} \rightarrow 0$ is meant to imply the smallest value of $z / \delta_{c}$ we can have without intruding into the buffer zone.

We will first look at the data of East, Sawyer \& Nash (1979) who produced a series 

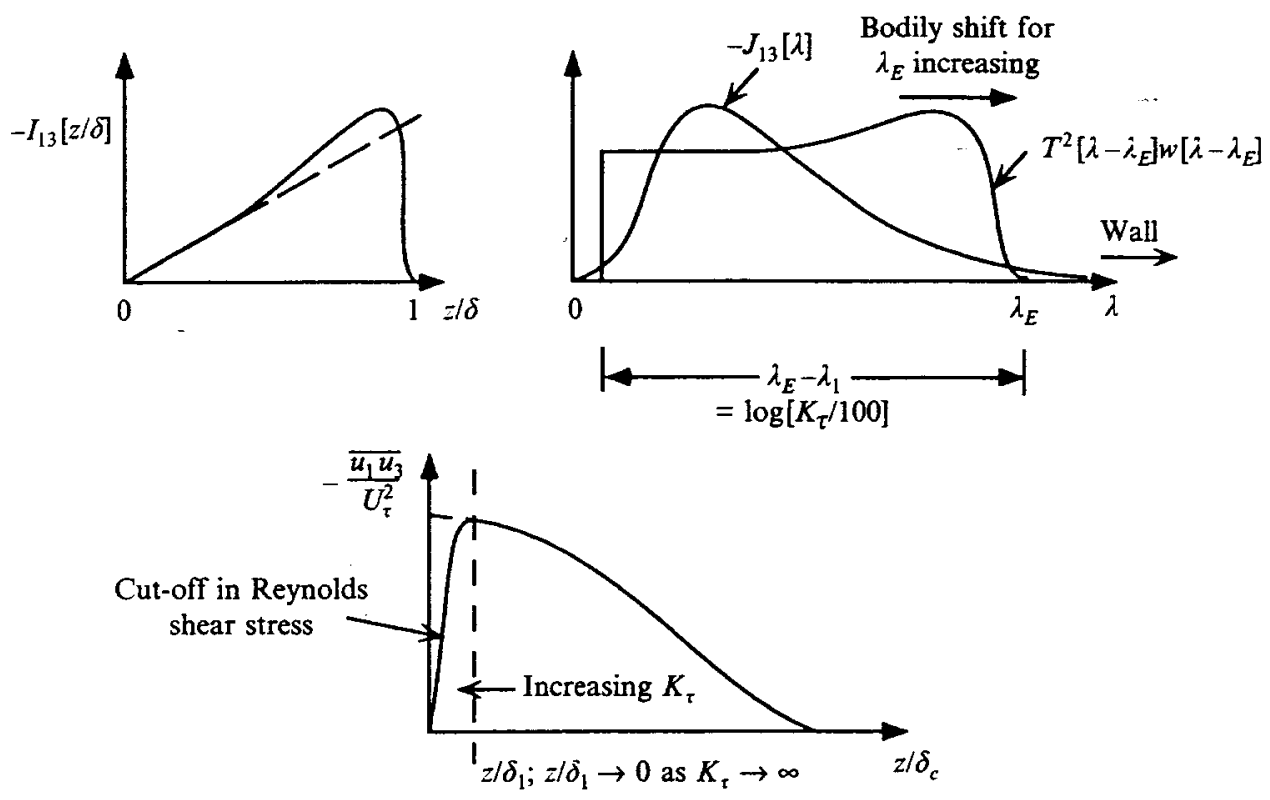

Figure 5. Effect of lower limit cut-off at $\lambda_{1}$.

East, Sawyer \& Nash (1979) data

$\begin{array}{cccccccccc}\text { Flow no. } & \text { symbol } & \Pi & S & \zeta & \beta & \sigma & H & R_{\theta} & K_{\tau} \\ 1 & \triangleleft & 0.34 & 26.7 & 0.09 & -0.25 & -0.14 & 1.31 & 7410 & 3424 \\ 2 & \triangleright & 0.41 & 27.3 & \approx 0.0 & -0.15 & \approx 0.0 & 1.29 & 10700 & 4563 \\ 3 & \triangle & 0.59 & 28.6 & -0.04 & -0.01 & 0.04 & 1.31 & 13000 & 4850 \\ 4 & \diamond & 0.95 & 30.9 & -0.18 & 0.47 & 0.08 & 1.35 & 18900 & 5757 \\ 5 & \square & 1.69 & 34.4 & -0.08 & 1.90 & 0.04 & 1.45 & 26800 & 6171 \\ 6 & 0 & 3.58 & 43.0 & 3.59 & 7.27 & -0.18 & 1.68 & 34700 & 5338\end{array}$

Skåre \& Krogstad (1994)

$$
\begin{array}{cccccccccc}
x(\mathrm{~m}) & \text { symbol } & \Pi & S & \zeta & \beta & \sigma & H & R_{\theta} & K_{\tau} \\
4.80 & \square & 6.85 & 59.4 & \approx 0.0 & 19.0 & \approx 0.0 & 1.99 & 49180 & 5123
\end{array}
$$

TABLE 1. Mean flow parameters for the equilibrium flow studies of East et al. (1979) and Skåre \& Krogstad (1994). (Flow no. 7 of East et al. $(\Pi=11.6)$ is not considered here since the turbulence intensities would have been too high for conventional hot-wire anemometry). Here $\sigma$ given by (16), $H=\delta^{*} / \theta, R_{\theta}=\theta U_{1} / v$ and $K_{\mathrm{\tau}}=\delta_{\tau} U_{\tau} / v ; \theta$ is the momentum thickness and $\delta^{*}$ is the displacement thickness.

of equilibrium boundary layers each having an approximately constant $\Pi$. Table 1 shows the important features of the data and $\sigma$ can be seen to be small, thus showing that the layers were either equilibrium layers or else quasi-equilibrium layers (only one velocity profile as published for each flow case).

The first eddy shape to test is the ' $\Pi$-shaped' eddy, the precise geometry of which is given in figure 6 . Now from the experiment, the mean-flow data provides all the necessary information for computing $\tau / \tau_{0}$ from equation (15), i.e. we require $S, \Pi, \beta$ and $\zeta$ as given in table 1. Equation (15) and shear stress data are compared in figure 7 and agreement appears to be reasonable although the flow might be slightly three- 

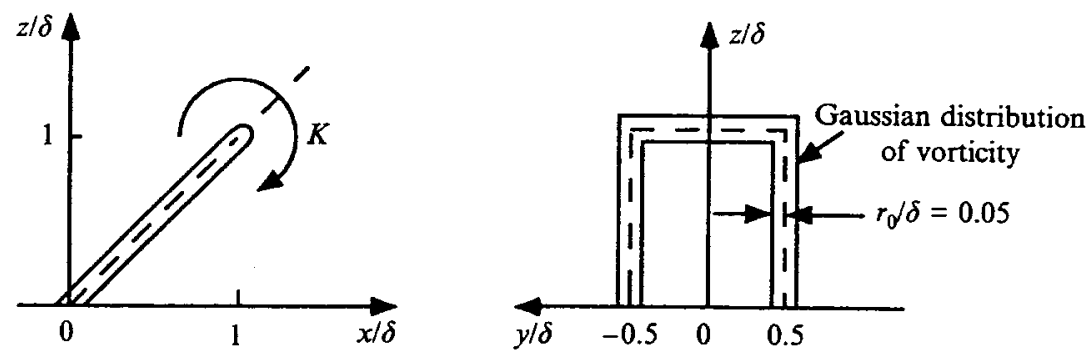

Figure 6. Details of type-A ' $\Pi$-shaped' eddy. Here $r_{0}$ is the standard deviation of the Gaussian distribution.

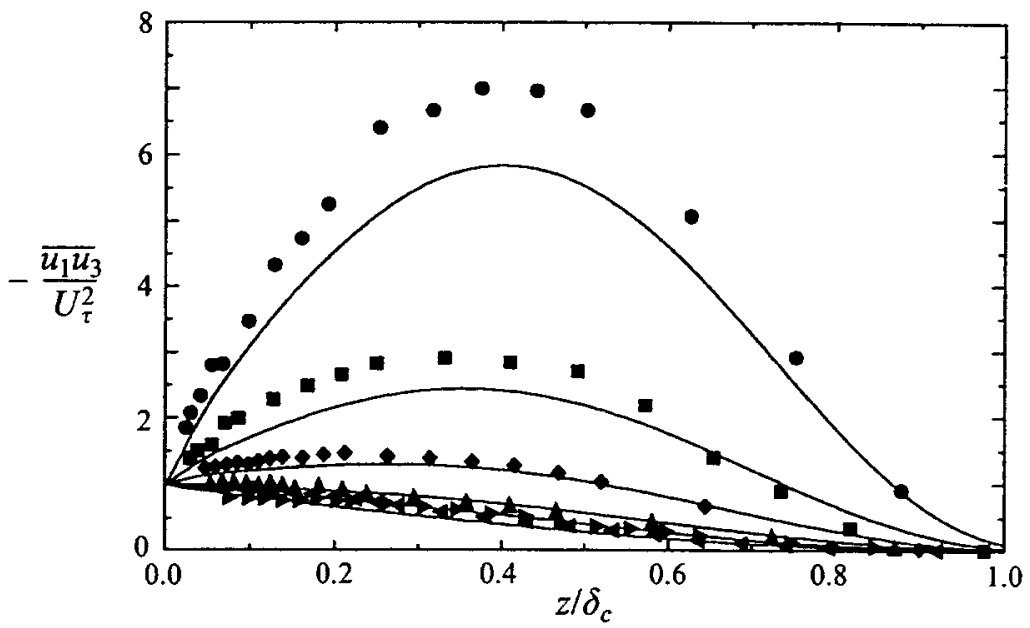

FIGURE 7. Reynolds shear stress data of the 'equilibrium' flow study of East et al. (1979) compared to formulation (15). (Symbols as given in table 1).

dimensional for $\Pi=3.58$. (Much better agreement will be seen with the authors' data in Part 2.)

Since the eddy shape is fixed, $J_{13}$ is known and since $\tau / \tau_{0}\left(\approx-\overline{u_{1} u_{3}} / U_{\tau}^{2}\right)$ is known from (15), equation (36) can be used to determine $T^{2}\left[\lambda-\lambda_{E}\right] w\left[\lambda-\lambda_{E}\right]$ by deconvolution for the various streamwise stations. For the purposes of deconvolution, a Fourier transform method was used which essentially involved the division of two Fourier transforms. The ends of the functions being deconvolved were slightly modified to avoid singularities or indeterminacies. Only smooth results from the deconvolution were accepted and were always tested by a convolution to make sure we could reconstruct the original function. Problems with this are discussed in $\S 8$. Once $T^{2}\left[\lambda-\lambda_{E}\right] w\left[\lambda-\lambda_{E}\right]$ is known, equation (36) can be used to determine the various other Reynolds stresses by a direct convolution calculation, again by using equation (36).

Figure 8 shows the results compared with data and the agreement is poor. Although the curves appear to have the correct semi-logarithmic behaviour in figure $8(a)$ for $z / \delta_{c} \rightarrow 0$, the intercepts are wrong and the 'bump' in the data at $z / \delta_{c} \approx 0.5$ is missing in the theoretical curves. The agreement in figure $8(c)$ is very poor for the downstream profiles. Unfortunately there are no data for the theoretical curves given in figure $8(b)$.

Various other eddy shapes were tried besides the simple ' $\Pi$ ' eddy but comparisons always showed poor agreement. These attempts included varying the inclinations of 

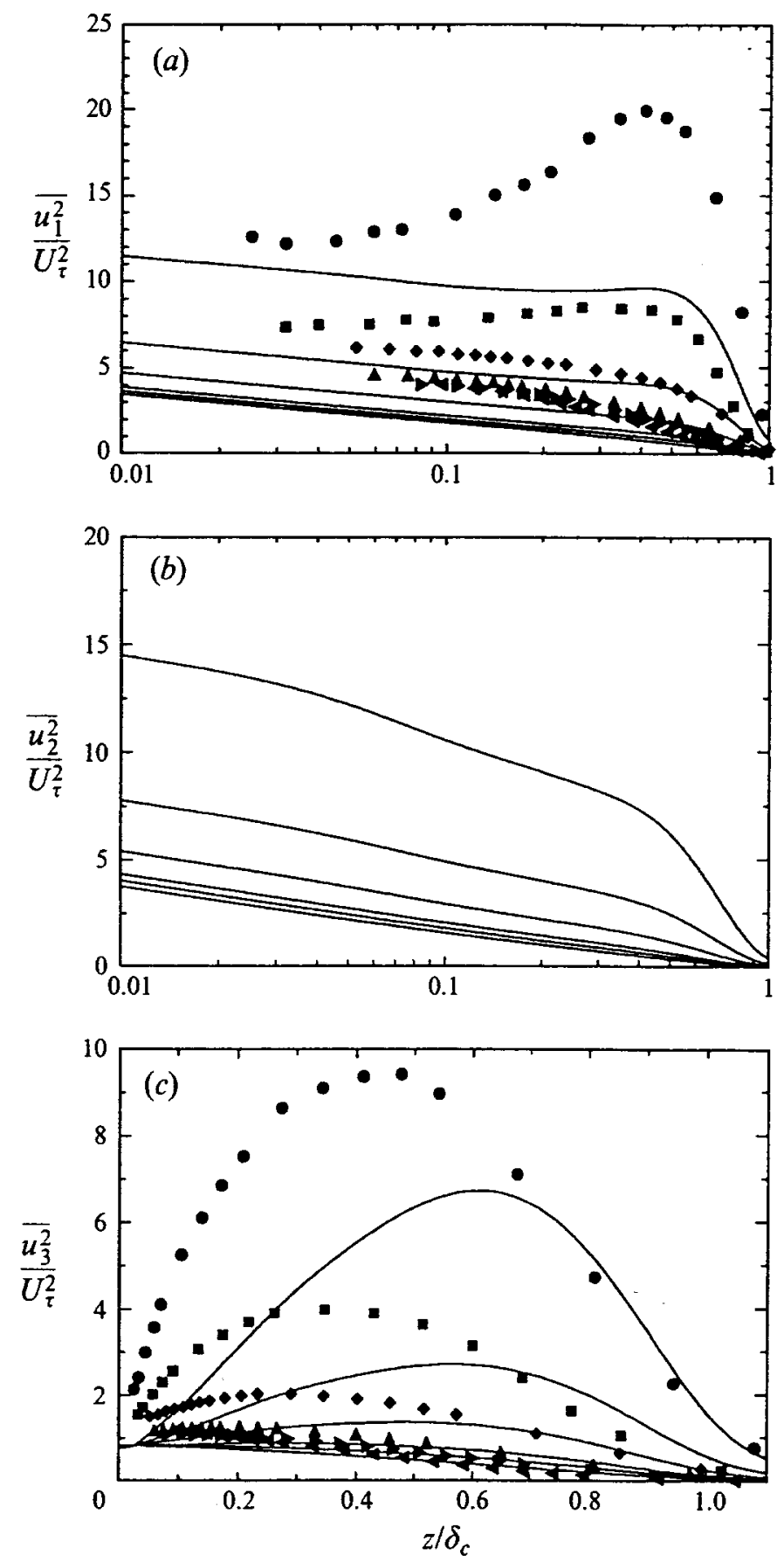

Eigure 8. Reynolds normal stresses for data of East et al. (1979) compared to formulation (36) asing a type-A ' $\Pi$-shaped' eddy structure alone. No spanwise measurements are available from East et al.

the eddies but this also did not help. The most important discrepancies are the absence of the 'bump' for high $\Pi$ values in $\overline{u_{1}^{2}} / U_{\tau}^{2}$ at $z / \delta_{c} \approx 0.5$ and incorrect intercepts. For low values of $\Pi$ the agreement was fair but as $\Pi$ increased, the agreement rapidly deteriorated. Clearly type-A eddies alone are inadequate no matter what their shape and some additional structure is needed. 


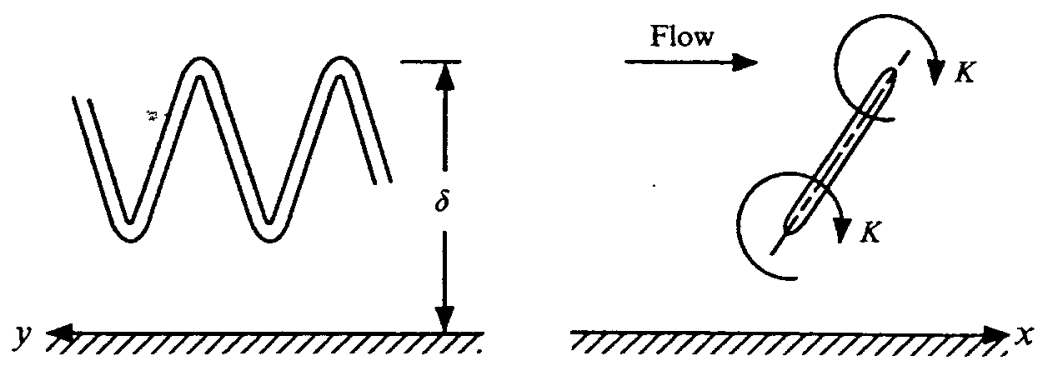

FIGURE 9. Sketch of typical type-B eddy.

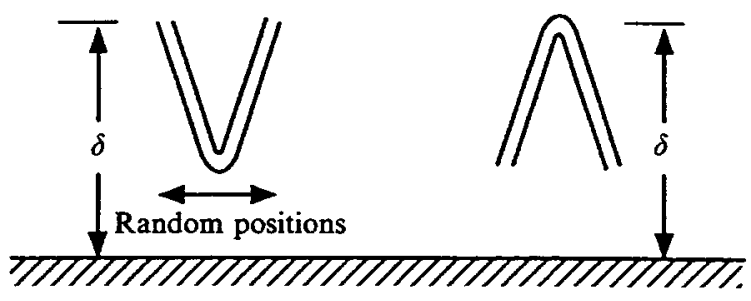

FIGURE 10. Simplified candidates to make up the type-B eddy structure.

\section{A second type of eddy structure (type-B)}

Considering the shortcomings of using type-A eddies alone, it is found that essentially what is needed are additional structures. Various types were tried including ring vortices but the only kind which gave the right kind of properties look like those shown in figure 9. Such structures will give the 'bump' needed in the $\overline{u_{1}^{2}} / U_{\tau}^{2}$ at $z / \delta_{c} \approx 0.5$ and we will refer to these as type-B structures.

Again we will assume a range of scales from $\delta_{1}$ to $\delta_{c}$ but the stresses are given by the sum of two convolution integrals

$$
\begin{aligned}
\left(\frac{\overline{u_{i} u_{j}}}{U_{\tau}^{2}}\right)_{A} & =\int_{-\infty}^{\infty} J_{i j_{A}}[\lambda] T_{A}^{2}\left[\lambda-\lambda_{E}\right] w_{A}\left[\lambda-\lambda_{E}\right] \mathrm{d} \lambda ; \\
\left(\frac{\overline{u_{i} u_{j}}}{U_{\tau}^{2}}\right)_{B} & =\int_{-\infty}^{\infty} J_{i j_{B}}[\lambda] T_{B}^{2}\left[\lambda-\lambda_{E}\right] w_{B}\left[\lambda-\lambda_{E}\right] \mathrm{d} \lambda ; \\
\frac{\overline{u_{i} u_{j}}}{U_{\tau}^{2}} & =\left(\frac{\overline{u_{i} u_{j}}}{U_{\tau}^{2}}\right)_{A}+\left(\frac{\overline{u_{i} u_{j}}}{U_{\tau}^{2}}\right)_{B}
\end{aligned}
$$

and immediately problems arise. The first problem is to obtain a random array of type-B eddies without an enormous computation effort. It was decided to replace them with ' $\Lambda$ '-type eddies as shown in figure 10 . This avoids the problem of having to jitter the spacings of adjacent undulations shown in figure 9 but it introduces vortex tubes which end in mid air. For the purposes of illustration here, the authors do not consider this to be a major shortcoming as the velocity field still remains divergence free even though this is not so locally for the vorticity field (see Winckelmans \& Leonard 1993). The position of the structures shown in figure 10 will be effectively randomized just as with type-A eddies by the use of the integrals given in $\S 3$.

The second problem is to decide how to partition the energies between the two sets of structures. Various schemes were attempted and all proved to be intractable. It was decided to try out an idea similar to one first proposed by Coles (1957). Let us 


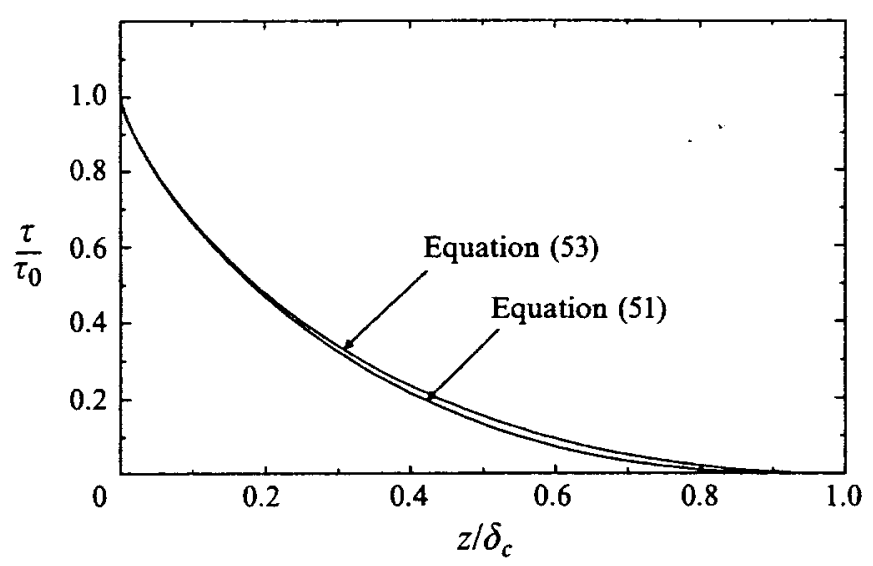

FIGURE 11. Sink flow shear stress profiles.

suppose that the turbulent boundary layer is made up of two sets of structures. One set are the 'wall structures' (type-A) and these contribute to the 'law of the wall' in the mean flow as well as in the turbulence structure and this structure is universal. In this flow $\Pi$ is zero. Such a flow exists in equilibrium sink flow (discussed in detail in Perry et al. 1994) and in fact it can be shown that $\beta=-0.5$ for $S \rightarrow \infty$. For finite $S$, we still have $\Pi$ very small and $\tau / \tau_{0}$ is very insensitive to $S$ provided the appropriate $\beta$ is used (see Perry et al. 1994). The shear stress distribution is shown in figure 11 and was calculated from (15) with $\Pi=0$. This is given by

$$
\frac{\tau}{\tau_{0}}=1-\frac{60}{59} \eta-\frac{20}{59} \eta^{3}+\frac{45}{59} \eta^{4}-\frac{24}{59} \eta^{5}+\frac{60}{59} \eta \log [\eta]
$$

'with the Lewkowicz (1982) formulation (9). This 'pure wall' flow (valid to $\eta=1$ ) is given by (1) and (9) as

$$
\frac{U}{U_{\tau}}=\frac{1}{\kappa} \log \left[\frac{z U_{\tau}}{v}\right]+A-\frac{1}{\kappa} \eta^{2}(1-\eta)(1-2 \eta) .
$$

This new extended 'law of the wall' is seen to include $\eta$ to ensure that $\mathrm{d} U / \mathrm{d} z=0$ at $\eta=1$. In earlier formulations only the logarithmic law of the wall was used and (51) reduces to

$$
\frac{\tau}{\tau_{0}}=1-\eta+\eta \log [\eta]
$$

which is close to (51) as seen in figure 11 . It can be seen that $T_{A}^{2}\left[\lambda-\lambda_{E}\right] w_{A}\left[\lambda-\lambda_{E}\right]$ is universal and can be determined by a deconvolution once type-A eddies are fixed.

The shear stress distribution in figure 11 is then subtracted from the total shear stress and once the type-B eddy geometry is fixed $T_{B}^{2}\left[\lambda-\lambda_{E}\right] w_{B}\left[\lambda-\lambda_{E}\right]$ can be found by a deconvolution. Hence once $T_{A}^{2}\left[\lambda-\lambda_{E}\right] w_{A}\left[\lambda-\lambda_{E}\right]$ and $T_{B}^{2}\left[\lambda-\lambda_{E}\right] w_{B}\left[\lambda-\lambda_{E}\right]$ are determined all other stress components and spectra can be found by convolution. All mathematical processes will be summarized in $\S$.

Regarding the mean flow, the type-B eddies will contribute to the Coles wake component. While this is very close to the idea of Coles, the only support we have for it here is that it is the only scheme that we have found so far that will work.

As mentioned earlier, equations (30) and (36) are decoupled and so the appropriate $T_{A}, T_{B}, w_{A}$ and $w_{B}$ can always be found to give the logarithmic law of the wall and the Coles law of the wake as well as the appropriate Reynolds stresses. 

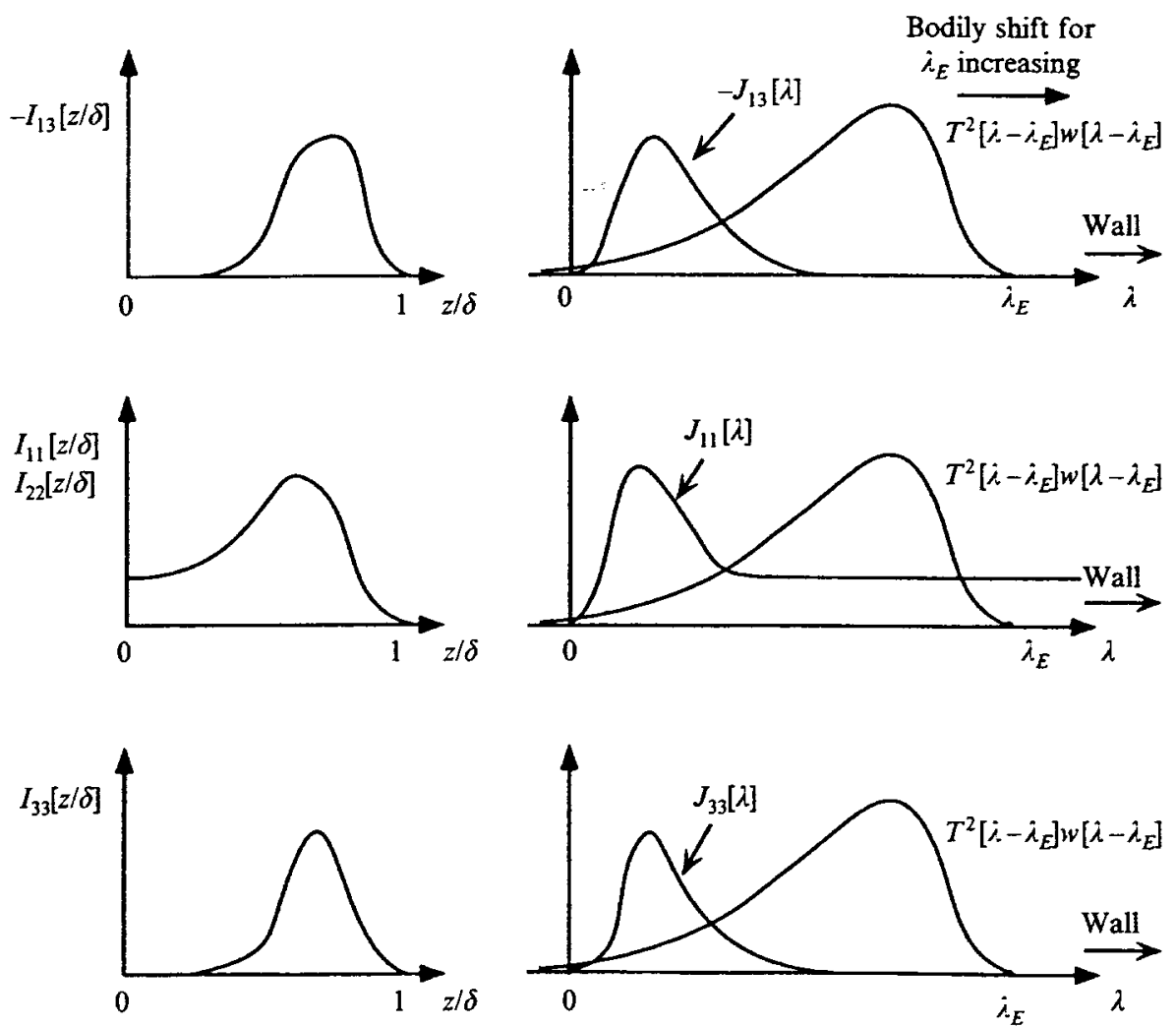

Figure 12. Typical eddy intensity functions for type-B eddies with $T^{2} w$ weighting functions.

\section{Mathematical properties of type-B eddies.}

Type- $B$ eddies are still attached eddies in the sense of Townsend since all aspects of their length scale are related to their height above the wall but their legs do not extend to the wall. They could perhaps be described as 'attached-detached' eddies. Numerical results show that the inviscid boundary condition at the wall is quite different from that given by type-A eddies. Firstly, $I_{13}$ is zero for a considerable range of $z / \delta, I_{11}$ and $I_{22}$ are constant for a considerable range of $z / \delta$ and $I_{33}$ is zero also over a considerable range as indicated in the sketch in figure 12. Actual $I_{i j}$ used for type-A and type-B eddies are shown in values (see $\S 8$ ). Deconvolutions show that $T^{2} w$ decreases to zero for sufficiently large negative values of $\lambda-\lambda_{E}$. From the figure it is obvious that for $\lambda_{E} \rightarrow \infty$ :

$$
-\frac{\overline{u_{1} u_{3}}}{U_{\tau}^{2}} \rightarrow 0, \frac{\overline{u_{3}^{2}}}{U_{\tau}^{2}} \rightarrow 0, \frac{\overline{u_{1}^{2}}}{U_{\tau}^{2}} \rightarrow \text { const. }=Q_{1} \text { (say) and } \frac{\overline{u_{2}^{2}}}{U_{\tau}^{2}} \rightarrow \text { const. }=Q_{2} \text { (say) }
$$

and this is applicable for a considerable range of $z / \delta_{c}$. Hence with both type-A and type-B eddies acting together we have for $\lambda_{E} \rightarrow \infty$

$$
\left.\begin{array}{rl}
-\overline{u_{1} u_{3}} / U_{\tau}^{2} & =1, \overline{u_{1}^{2}} / U_{\tau}^{2}=H_{1}+Q_{1}-A_{1} \log \left[z / \delta_{c}\right] \\
\overline{u_{2}^{2}} / U_{\tau}^{2} & =H_{2}+Q_{2}-A_{2} \log \left[z / \delta_{c}\right], \quad \overline{u_{3}^{2}} / U_{\tau}^{2}=K_{3} .
\end{array}\right\}
$$


Type-A eddy
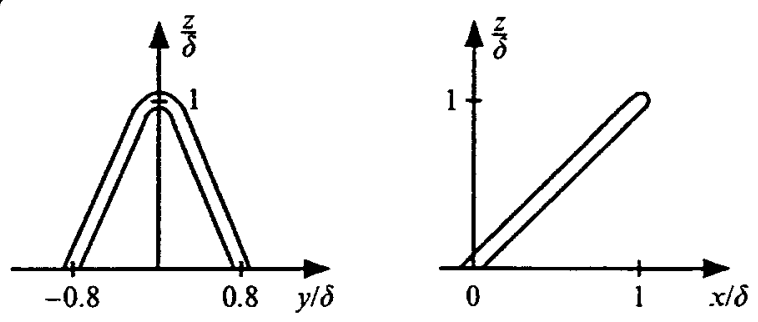

Type-B eddies (Ensemble)
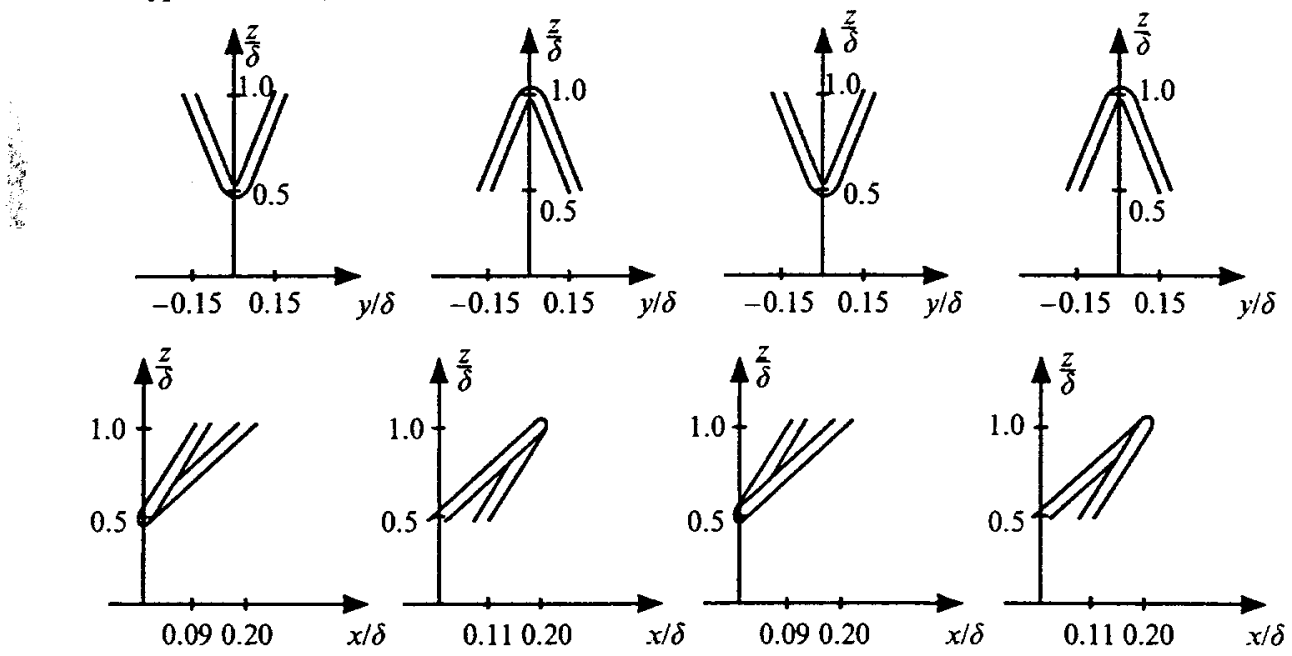

Figure 13. Type-A and type-B eddy structures chosen for calculation. The resulting type-B structure is an ensemblage of the four above structures. $r_{0} / \delta=0.05$ in all cases; Gaussian distribution of vorticity is assumed in the vortex tubes.

\section{Choice of eddy shapes and comparison of stresses with experiment}

The choice of geometry for type-A and type-B eddies at this stage must be based on trial and error. It is a very time consuming process and will remain so until some systematic search can be achieved (this will most probably require an increase in computational rates). Eddy shapes are presented here purely to prove the point that a single universal shape for type-A and type-B performs extremely well for all situations. The authors wish to emphasize that these are temporary shapes to be changed at any time without notice. The present candidates are shown in figure 14. It will be noted that type-B eddies are not contained in a $45^{\circ}$ plane orthogonal to the $(x, z)$-plane but are twisted out of the plane. Thus we have an ensemblage of left- and right-handed, upright $\Lambda$ 's and upside down $\Lambda$ 's constituting type-B eddies as shown in the figure. For the type-A eddies ' $\Lambda$ '-shaped eddies were chosen and are also shown in the figure. These shapes were chosen mainly to match $\overline{u_{2}^{2}} / U_{\tau}^{2}$ data of the authors given in Part 2. 'Twisted' type-A eddies have not been tried. For all eddy shapes chosen, we have attempted to use only long straight vortex segments. These have simple analytic solutions which make for rapid and efficient computations. Once these eddy geometries are chosen, the only information necessary is $\Pi, S, \beta$ and $\zeta$ which are substituted into equation (15). From this, all Reynolds stress distributions and spectra are produced from convolution integrals without any adjustment to any constants. Everything basically comes from the momentum equation (coupled with the mean-flow law of the wall and law of the wake) once the eddy geometries are fixed. 

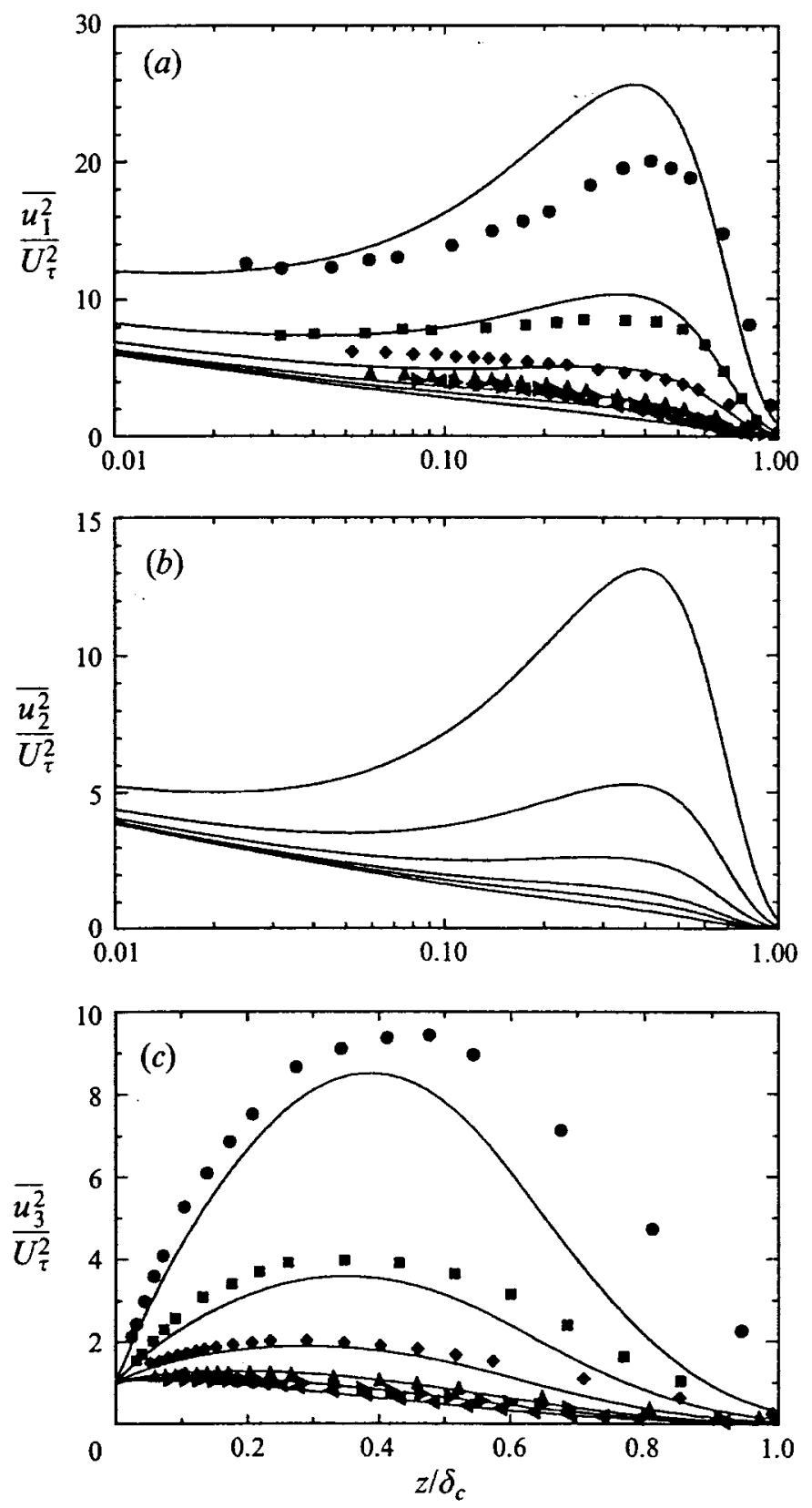

FIGURE 14. Reynolds normal stresses for data of East et al. (1979) compared to formulation (50) using type-A and type-B eddies as shown in figure 13. No spanwise measure:nents are available from East et al.

Figures $14(a), 14(b)$ and $14(c)$ show the profiles of all the normal stress components for the various flow cases of East et al. Although the agreement is not perfect, it is a great improvement over the agreement shown in figure 8 . As a matter of interest, using the theory outlined in $\S 3$, the actual eddy intensity functions computed for the assumed eddy shapes are given in Appendix C.

In the literature, measurements using modern hot-wire techniques of Reynolds shear stresses together with all three normal stresses for layers with pressure gradients are rare. Recently Skåre \& Krogstad (1994) produced such measurements for one equilibrium layer and details are shown in table 1. 


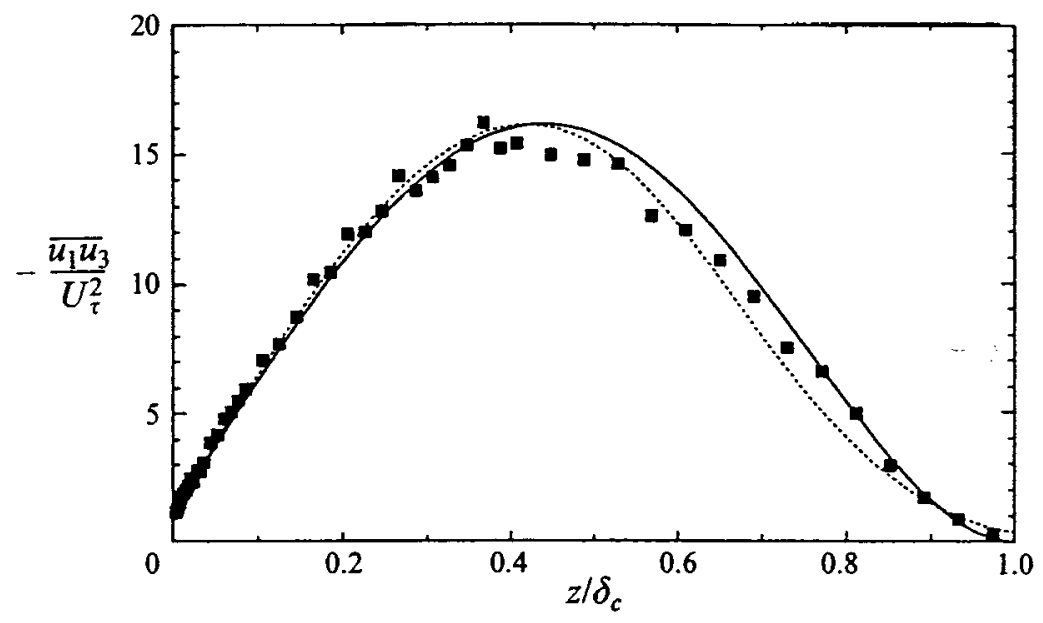

Figure 15. Reynolds shear stress data of the 'equilibrium' flow study of Skàre \& Krogstad (1994) tompared to formulation (15) (solid line). The broken line represents the reconstructioned profile after the deconvolution procedure. See text.

Figure 15 shows the Reynolds shear stress distribution computed from values given in the table using (15) and agreement can be seen to be reasonable. Comparisons with the three components of normal Reynolds stresses are given in figures $16(a)$, $16(b)$ and 16(c). Again agreement seems reasonable and it is obvious that using type- $\mathrm{A}$ and type-B eddies is a great improvement over type-A eddies alone. It will be noted that with the data of East et al. (1979) and Skåre \& Krogstad (1994) there appears to be a discrepancy with the theory for $\overline{u_{3}^{2}} / U_{\tau}^{2}$ in the outer part of the flow. The authors believe that this is partly due to inaccuracies in the computational technique developed. A modified Fourier transform deconvolution method has been used and the reconstructed $-\overline{u_{1} u_{3}} / U_{\tau}^{2}$ distribution after the $T^{2} w$ distribution has been established never quite agrees with the original distribution as given by (15). An example of this is shown in figure 15 as a broken curve. Any attempt to obtain a better match often led to instabilities in the calculations. Since these discrepancies are minor, particularly in the authors' data to be reported in Part 2, this numerical problem was not pursued.

\section{Summary of computational methods}

Figure 17 shows an overview of the convolution and deconvolution calculations carried out if we assume that the turbulent boundary layer consists of type-A eddies alone. It can be seen that this will give all Reynolds stresses provided we know $\Pi$, $S, \beta$ and $\zeta$ and the eddys shape, i.e. the $J_{i j}[\lambda]$. Figure 18 shows a flowchart for the corresponding calculations for the wall-wake eddy structure model.

\section{Conclusions}

Here a modification of the attached eddy hypothesis has been made in order to explain data for boundary layers with pressure gradient. Comparisons with the data of East et al. (1979) and Skåre \& Krogstad (1994) show encouraging agreement with this modified hypothesis. Unlike previous versions, it was found necessary to incorporate both 'wall structure' (type-A eddies) and 'wake structure' (type-B eddies) into the model. 

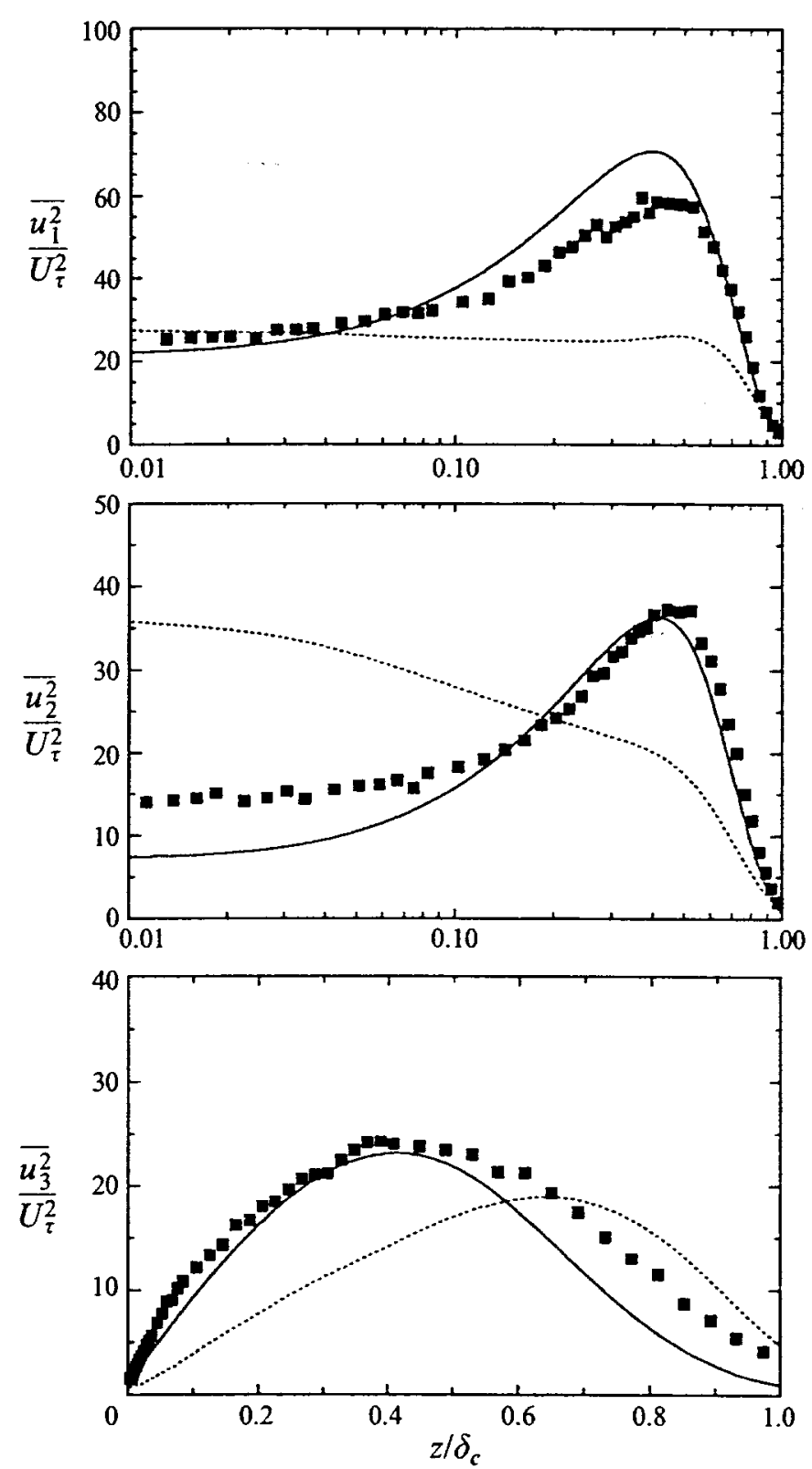

FIGURE 16. Reynolds normal stresses for data of Skåre \& Krogstad (1994) compared to formulation (50) using type-A and type-B eddies as shown in figure 13. Broken line is for type-A eddies alone.

The data tested here are from equilibrium and quasi-equilibrium layers. In Part 2, an extensive set of non-equilibrium data produced by the authors has been compared with this extended attached eddy hypothesis.

The authors wish to thank the Australian Research Council for the financial support of this project. We acknowledge the Fairchild Foundation for supporting the senior author as a Fairchild Scholar at Caltech in 1992 where he developed some of the analysis. Also we acknowledge the many interesting and fruitful discussions with Professor D. Coles at GALCIT, Caltech and Professor P.-A. Krogstad of the Norwegian Institute of Technology for kindly providing experimental data. 


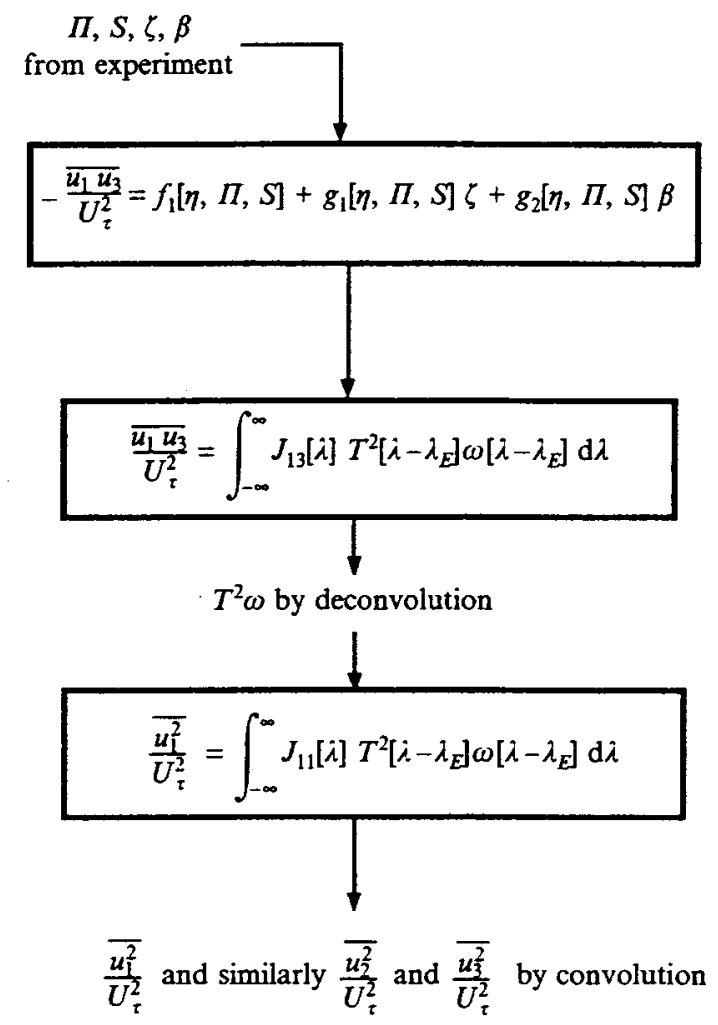

FIGURE 17. Overview of calculations for type-A eddy structures alone.

\section{Appendix A}

Substituting (2) and (3) into (4) and integrating we obtain

$$
\frac{\tau}{\tau_{0}}=1+A_{1} X_{1}+A_{2} X_{2}+A_{3} X_{3}+A_{4} X_{4}
$$

where

$$
A_{i}=A_{i}[\eta, \Pi, S]
$$

and

$$
X_{1}=\frac{\mathrm{d} \delta_{c}}{\mathrm{~d} x}, \quad X_{2}=\frac{\delta_{c}}{S} \frac{\mathrm{d} S}{\mathrm{~d} x}, \quad X_{3}=\delta_{c} \frac{\mathrm{d} \Pi}{\mathrm{d} x}, \quad X_{4}=\frac{\delta_{c}}{U_{1}} \frac{\mathrm{d} U_{1}}{\mathrm{~d} x} .
$$

The $A_{i}$ are given by

$$
\begin{aligned}
& A_{1}=\int_{0}^{\eta} f^{2} \mathrm{~d} \eta-f \int_{0}^{\eta} f \mathrm{~d} \eta+S\left(\eta f-\int_{0}^{\eta} f \mathrm{~d} \eta\right), \\
& A_{2}=-2 \int_{0}^{\eta} f^{2} \mathrm{~d} \eta+f \int_{0}^{\eta} f \mathrm{~d} \eta+S \int_{0}^{\eta} f \mathrm{~d} \eta \\
& A_{3}=\frac{\mathrm{d}}{\mathrm{d} \Pi} \int_{0}^{\eta} f^{2} \mathrm{~d} \eta-(f+S) \frac{\mathrm{d}}{\mathrm{d} \Pi} \int_{0}^{\eta} f d \eta \\
& A_{4}=2 \int_{0}^{\eta} f^{2} \mathrm{~d} \eta-f \int_{0}^{\eta} f \mathrm{~d} \eta+S\left(\eta f-3 \int_{0}^{\eta} f \mathrm{~d} \eta\right) .
\end{aligned}
$$

This is the form arrived at by Perry \& $\mathrm{Li}(1991)$ and in an equivalent form by Marušic (1991). Now relationships can be established between the $X_{i}$ as follows. From the 
Wall Structure

Wake Structure

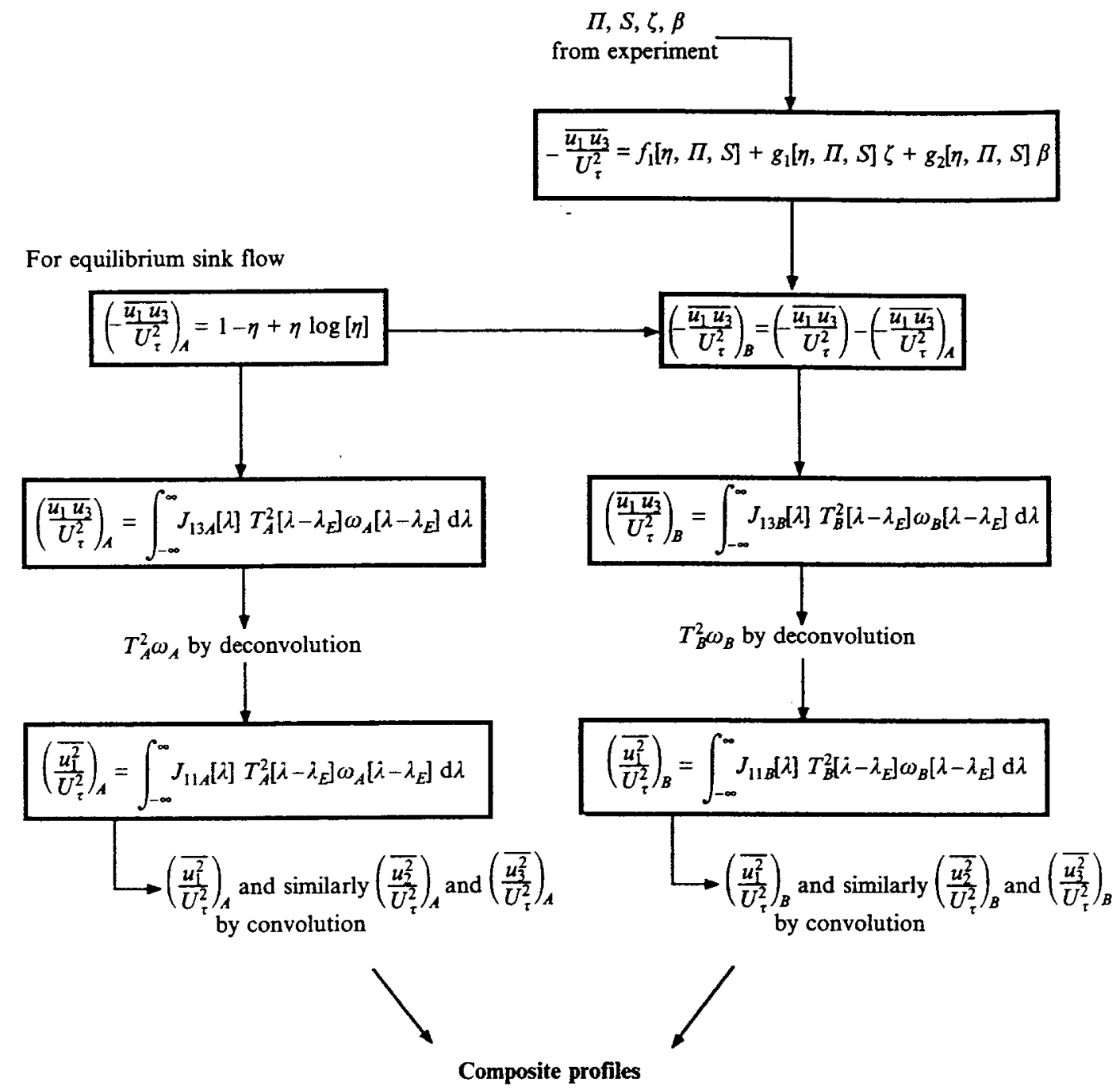

FIGURE 18. Overview of calculations for the wall-wake eddy structure model.

logarithmic law of the wall and law of the wake

$$
X_{2}=E_{1}\left(X_{1}+N X_{3}+X_{4}\right)
$$

where

and

$$
E_{1}=\frac{1}{\kappa S+1}
$$

$$
N=W_{c}[1, \Pi]+\Pi \frac{\mathrm{d} W_{c}[1, \Pi]}{\mathrm{d} \Pi} .
$$

From the outer boundary condition $\tau / \tau_{0}=0$ at $\eta=1$

$$
0=1+B_{1} X_{1}+B_{2} X_{2}+B_{3} X_{3}+B_{4} X_{4}
$$

where

$$
B_{i}=A_{i}[1, \Pi, S]
$$


From (A 3)-(A 6) $X_{1}$ and $X_{2}$ can be expressed in terms of $X_{3}$ and $X_{4}$ to give

where

$$
\frac{\tau}{\tau_{0}}=f_{1}[\eta, \Pi, S]+f_{2}[\eta, \Pi, S] \delta_{c} \frac{\mathrm{d} \Pi}{\mathrm{d} x}+f_{3}[\eta, \Pi, S] \frac{\delta_{c}}{U_{1}} \frac{\mathrm{d} U_{1}}{\mathrm{~d} x}
$$

$$
\begin{aligned}
f_{1}[\eta, \Pi, S]=1- & A_{1}[\eta, \Pi, S] /\left(B_{1}[\Pi, S]\right. \\
+ & \left.E_{1} B_{2}[\Pi, S]\right)-E_{1} A_{2}[\eta, \Pi, S] /\left(B_{1}[\Pi, S]+E_{1} B_{2}[\Pi, S]\right), \\
f_{2}[\eta, \Pi, S]= & \left(E_{1} \mathrm{~N} A_{2}[\eta, \Pi, S] B_{1}[\Pi, S]\right. \\
& +A_{3}[\eta, \Pi, S] B_{1}[\Pi, S]-E_{1} \mathrm{~N} A_{1}[\eta, \Pi, S] B_{2}[\Pi, S] \\
& +E_{1} A_{3}[\eta, \Pi, S] B_{2}[\Pi, S]-A_{1}[\eta, \Pi, S] B_{3}[\Pi, S] \\
& \left.-E_{1} A_{2}[\eta, \Pi, S] B_{3}[\Pi, S]\right)\left(B_{1}[\Pi, S]+E_{1} B_{2}[\Pi, S]\right), \\
f_{3}[\eta, \Pi, S]= & \left(E_{1} A_{2}[\eta, \Pi, S] B_{1}[\Pi, S]\right. \\
& +A_{4}[\eta, \Pi, S] B_{1}[\Pi, S]-E_{1} A_{1}[\eta, \Pi, S] B_{2}[\Pi, S] \\
& +E_{1} A_{4}[\eta, \Pi, S] B_{2}[\Pi, S]-A_{1}[\eta, \Pi, S] B_{4}[\Pi, S] \\
& \left.-E_{1} A_{2}[\eta, \Pi, S] B_{4}[\Pi, S]\right) /\left(B_{1}[\Pi, S]+E_{1} B_{2}[\Pi, S]\right),
\end{aligned}
$$

$f_{1}[\eta, \Pi, S]=\left(-S e_{1}[1, \Pi]+E_{1} S e_{1}[1, \Pi]+S e_{1}[\eta, \Pi]\right.$

$-E_{1} S e_{1}[\eta, \Pi]+e_{2}[1, \Pi]-2 E_{1} e_{2}[1, \Pi]-e_{2}[\eta, \Pi]+2 E_{1} e_{2}[\eta, \Pi]$

$-e_{3}[1, \Pi]+E_{1} e_{3}[1, \Pi]+e_{3}[\eta, \Pi]-E_{1} e_{3}[\eta, \Pi]$

$\left.+S e_{4}[1, \Pi]-S e_{4}[\eta, \Pi]\right) /\left(-S e_{1}[1, \Pi]+E_{1} S e_{1}[1, \Pi]+e_{2}[1, \Pi]\right.$

$\left.-2 E_{1} e_{2}[1, \Pi]-e_{3}[1, \Pi]+E_{1} e_{3}[1, \Pi]+S e_{4}[1, \Pi]\right)$,

$$
\begin{aligned}
f_{2}[\eta, \Pi, S]= & \left(E _ { 1 } \mathrm { N } ( S e _ { 1 } [ \eta , \Pi ] - 2 e _ { 2 } [ \eta , \Pi ] + e _ { 3 } [ \eta , \Pi ] ) \left(e_{2}[1, \Pi]-e_{3}[1, \Pi]\right.\right. \\
& \left.+S\left(-e_{1}[1, \Pi]+e_{4}[1, \Pi]\right)\right)-E_{1} \mathrm{~N}\left(S e_{1}[1, \Pi]-2 e_{2}[1, \Pi]\right. \\
& \left.+e_{3}[1, \Pi]\right)\left(e_{2}[\eta, \Pi]-e_{3}[\eta, \Pi]+S\left(-e_{1}[\eta, \Pi]\right.\right. \\
& \left.\left.+e_{4}[\eta, \Pi]\right)\right)-E_{1}\left(S e_{1}[\eta, \Pi]-2 e_{2}[\eta, \Pi]+e_{3}[\eta, \Pi]\right)\left(-\left(S e_{5}[1, \Pi]\right)\right. \\
& \left.+e_{6}[1, \Pi]-e_{7}[1, \Pi]\right)-\left(e_{2}[\eta, \Pi]-e_{3}[\eta, \Pi]+S\left(-e_{1}[\eta, \Pi]\right.\right. \\
& \left.\left.+e_{4}[\eta, \Pi]\right)\right)\left(-\left(S e_{5}[1, \Pi]\right)+e_{6}[1, \Pi]-e_{7}[1, \Pi]\right) \\
& +E_{1}\left(S e_{1}[1, \Pi]-2 e_{2}[1, \Pi]+e_{3}[1, \Pi]\right)\left(-\left(S e_{5}[\eta, \Pi]\right)\right. \\
& \left.+e_{6}[\eta, \Pi]-e_{7}[\eta, \Pi]\right)+\left(e_{2}[1, \Pi]-e_{3}[1, \Pi]+S\left(-e_{1}[1, \Pi]\right.\right. \\
& \left.\left.\left.+e_{4}[1, \Pi]\right)\right)\left(-\left(S e_{5}[\eta, \Pi]\right)+e_{6}[\eta, \Pi]-e_{7}[\eta, \Pi]\right)\right) /\left(e_{2}[1, \Pi]-e_{3}[1, \Pi]\right. \\
& \left.+E_{1}\left(S e_{1}[1, \Pi]-2 e_{2}[1, \Pi]+e_{3}[1, \Pi]\right)+S\left(-e_{1}[1, \Pi]+e_{4}[1, \Pi]\right)\right)
\end{aligned}
$$

$f_{3}[\eta, \Pi, S]=\left(-S e_{1}[\eta, \Pi] e_{2}[1, \Pi]+3 E_{1} S e_{1}[\eta, \Pi] e_{2}[1, \Pi]\right.$

$+S e_{1}[1, \Pi] e_{2}[\eta, \Pi]-3 E_{1} S e_{1}[1, \Pi] e_{2}[\eta, \Pi]$

$+2 S e_{1}[\eta, \Pi] e_{3}[1, \Pi]-2 E_{1} S e_{1}[\eta, \Pi] e_{3}[1, \Pi]-e_{2}[\eta, \Pi] e_{3}[1, \Pi]$

$+E_{1} e_{2}[\eta, \Pi] e_{3}[1, \Pi]-2 S e_{1}[1, \Pi] e_{3}[\eta, \Pi]+2 E_{1} S e_{1}[1, \Pi] e_{3}[\eta, \Pi]$

$+e_{2}[1, \Pi] e_{3}[\eta, \Pi]-E_{1} e_{2}[1, \Pi] e_{3}[\eta, \Pi]-2 S^{2} e_{1}[\eta, \Pi] e_{4}[1, \Pi]$

$\left.+S e_{2}[\eta, \Pi] e_{4}[1, \Pi]+2 S^{2} e_{1}[1, \Pi] e_{4}[\eta, \Pi]-S e_{2}[1, \Pi] e_{4}[\eta, \Pi]\right) /$

$\left(-S e_{1}[1, \Pi]+E_{1} S e_{1}[1, \Pi]+e_{2}[1, \Pi]-2 E_{1} e_{2}[1, \Pi]-e_{3}[1, \Pi]\right.$

$\left.+E_{1} e_{3}[1, \Pi]+S e_{4}[1, \Pi]\right)$ 


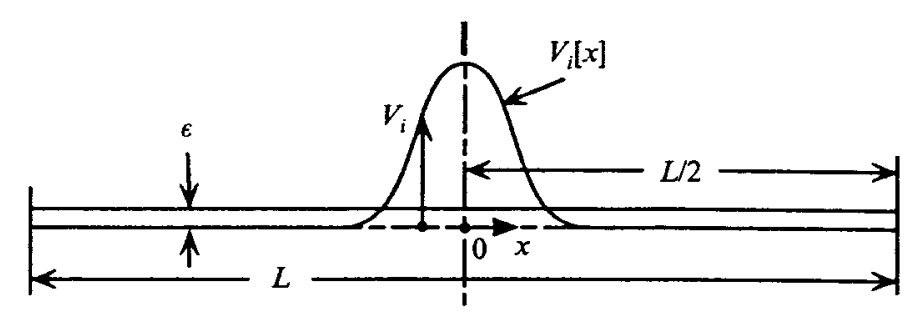

FIGURE 19. Typical velocity signature over interval $L$

where

$$
\left.\begin{array}{l}
e_{1}=\int_{0}^{\eta} f \mathrm{~d} \eta, e_{2}=\int_{0}^{\eta} f^{2} \mathrm{~d} \eta, e_{3}=f \int_{0}^{\eta} f \mathrm{~d} \eta, e_{4}=\eta f, \\
e_{5}=\frac{\mathrm{d}}{\mathrm{d} \Pi} \int_{0}^{\eta} f \mathrm{~d} \eta, e_{6}=\frac{\mathrm{d}}{\mathrm{d} \Pi} \int_{0}^{\eta} f^{2} \mathrm{~d} \eta \text { and } e_{7}=f \frac{\mathrm{d}}{\mathrm{d} \Pi} \int_{0}^{\eta} f \mathrm{~d} \eta
\end{array}\right\}
$$

\section{Appendix B}

The velocity components $V_{i}$ used in equation (32) obtained from the Biot-Savart law are the contributions made by an isolated eddy and its image in the wall. This velocity is relative to an observer at rest with the fluid at infinity and the convection velocity of the eddy does not need to be specified in this calculation. Figure 19 shows a typical $V_{i}$ pulse and it is of a limited spatial length.

In the figure $L$ is the length over which we wish to obtain an average $\epsilon$, and this is by definition

$$
\epsilon=\frac{1}{L} \int_{-L / 2}^{L / 2} V_{i} \mathrm{~d} x
$$

and this applies also in the limit as $L \rightarrow \infty$ in which case $\epsilon \rightarrow 0$.

Suppose we have a spatially periodic signal with a pulse of shape $V_{i}$ repeated with the spacing of length $L$, then

$$
V_{i}=v_{i}+\epsilon
$$

where $v_{i}$ is a perturbation with zero mean and

$$
\frac{1}{L} \int_{-L / 2}^{L / 2} v_{i}^{2} \mathrm{~d} x=\frac{1}{L} \int_{-L / 2}^{L / 2} V_{i}^{2} \mathrm{~d} x-\epsilon^{2} .
$$

However, it can be shown that if $V_{i}$ decays faster than $x^{-1}$ for $x \rightarrow \infty$, then

$$
\lim _{L \rightarrow \infty} \frac{\epsilon^{2}}{\frac{1}{L} \int_{-L / 2}^{L / 2} V_{i}^{2} \mathrm{~d} x}=0
$$

Hence $V_{i}$, the Biot-Savart law signature, can be used for computing mean squares and cross-correlations of the fluctuating velocity components which have a zero mean. By way of example, suppose that a zero mean signal $S(x)$ is made up of a large number $n$ of pulses given by $V_{i}$ with random phases within the interval $L$. For infinite $L, n$ also approaches infinity and the mean square of $S$ is given by

$$
\overline{S^{2}}=\lim _{L \rightarrow \infty} \frac{n}{L} \int_{-L / 2}^{L / 2} V_{i}^{2} \mathrm{~d} x=\frac{1}{\delta_{x}} \int_{-\infty}^{\infty} V_{i}^{2} \mathrm{~d} x
$$



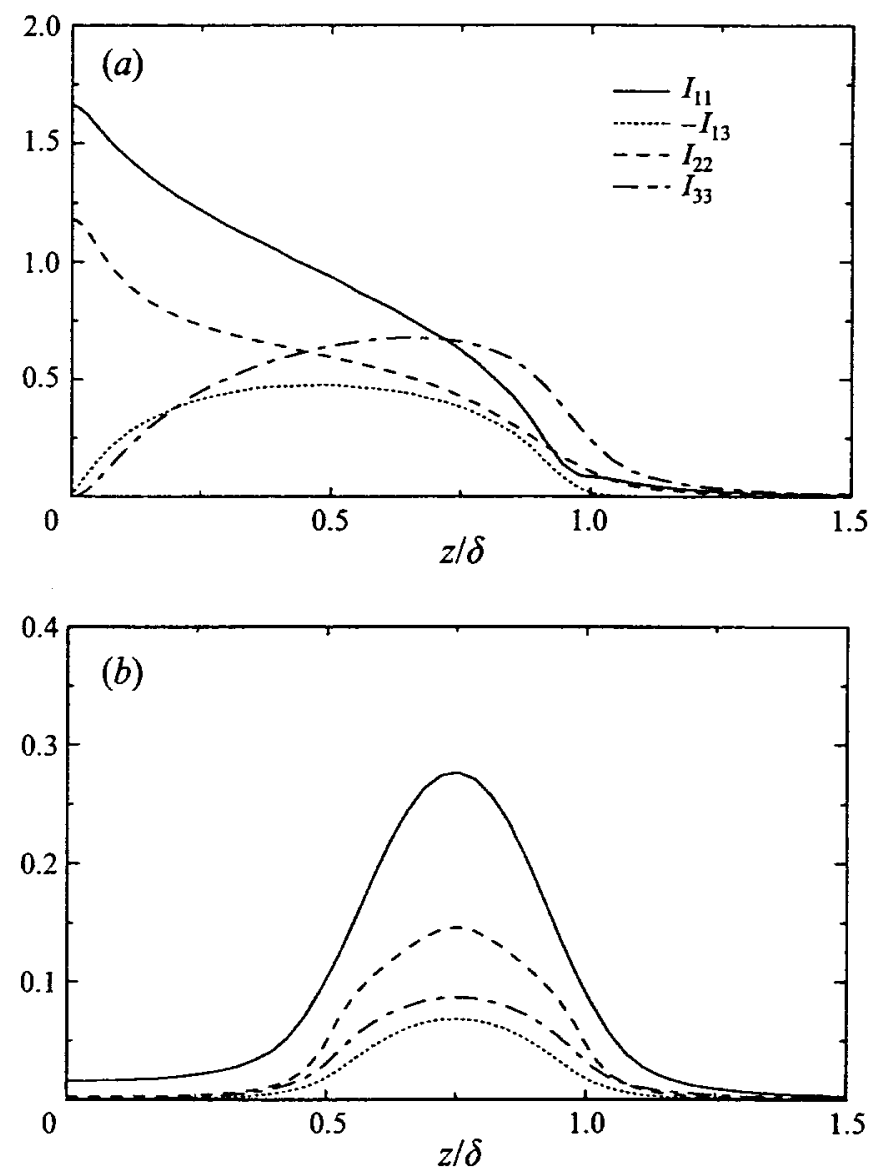

FIGURE 20. (a) Type-A eddy intensity functions, (b) Type-B eddy intensity functions

where $\delta_{x}$ is the average spacing of pulses. This forms the basis of analysis given in $\S 3$ where the concept has been extended to two-dimensional distributions.

\section{Appendix C}

Figures $20(a)$ and $20(b)$ show computed eddy intensity functions for the type-A and type-B eddies shown in figure 13. The results are normalized here such that $T_{A}^{2}\left[\lambda-\lambda_{E}\right] w_{A}\left[\lambda-\lambda_{E}\right]=1$ for $\left(\lambda-\lambda_{E}\right) \rightarrow-\infty$.

\section{REFERENCES}

Clauser, F. H. 1954 Turbulent boundary layers in adverse pressure gradients. J. Aero. Sci. 21, 91-108.

Clauser, F. H. 1956 The turbulent boundary layer. Adv. Appl. Mech. 4, 1-51.

Coles, D. E. 1956 The law of the wake in the turbulent boundary layer. J. Fluid Mech. 1, 191-226.

COLES, D. E. 1957 Remarks on the equilibrium turbulent boundary layer. J. Aero. Sci. 24, 459-506.

EAST, L. F., SAWYER, W. G. \& NASH, C. R. 1979 An investigation of the structure of equilibrium turbulent boundary layers. RAE Tech. Rep. 79040.

LEWKowICZ, A. K. 1982 An improved universal wake function for turbulent boundary layers and some of its consequences. Z. Flugwiss. Weltraumforsch. 6, 261-266.

MARUŠí́, I. 1991 The structure of zero- and adverse- pressure gradient turbulent boundary layers. $\mathrm{PhD}$ thesis, University of Melbourne. 
MARUŠÍ́, I. \& PERRY, A. E. 1995 A wall-wake model for the turbulence structure of boundary layers. Part 2. Further experimental support. J. Fluid Mech. 298, 389-407.

PERry, A. E. 1968 A theoretical study of equilibrium turbulent boundary layers. Originally unpublised; now, Rep. FM-19, 1992 Dept. of Mech. Engng, University of Melbourne.

PERRY, A. E. 1992 A new look at some closure problems of turbulent boundary layers. GALCIT Rep. FM-92-4. California Institute of Technology.

Perry, A. E. \& Chong, M. S. 1982 On the mechanism of wall turbulence. J. Fluid Mech. 119, 173-217.

Perry, A. E., Henbest, S. M. \& Chong, M. S. 1986 A theoretical and experimental study of wall turbulence. J. Fluid Mech. 165, 163-199.

Perry, A. E. \& Ll, J. D. 1990 Experimental support for the attached eddy hypothesis in zeropressure-gradient turbulent boundary layers. J. Fluid Mech. 218, 405-438.

Perry, A. E. \& LI, J. D. 1991 Theoretical and experimental studies of shear stress profiles in two dimensional turbulent boundary layers. Rep. FM-18. Dept. of Mech. Engng, University of Melbourne.

PERry, A. E., LI, J. D. \& MARušíc, I. 1991 Towards a closure scheme for turbulent boundary layers using the attached eddy hypothesis. Phil Trans. R. Soc. Lond. A 336, 67-79.

Perry, A. E., LIM, K. L. \& Henbest, S. M. 1987 An experimental study of the turbulence structure in smooth- and rough-wall boundary layers. J. Fluid Mech. 177, 437-466.

Perry, A. E., MARušić, I. \& LI, J. D. 1993 A wall-wake model for the turbulence structure of boundary layers using attached eddies. Rep. FM-21. Dept. of Mech. Engng, University of Melbourne.

Perry, A. E., Marušić, I. \& LI, J. D. 1994 Wall turbulence closure based on classical similarity laws and the attached eddy hypothesis. Phys. Fluids 6, 1024-1035.

RotTA, J. C. 1962 Turbulent boundary layers in incompressible flow. Prog. Aero. Sci. 2, 1-219.

SKÅR, P. E. \& KRogSTAD, P.-A. 1994 A turbulent boundary layer near separation. J. Fluid Mech. 272, 319-348.

Townsend, A. A. 1961 Equilibrium layers and wall turbulence. J. Fluid Mech. 11, 97-120.

Townsend, A. A. 1976 The Structure of Turbulent Shear Flow. Cambridge University Press.

Winckelmans, G. S. \& LeONARD, A. 1993 Contributions to vortex particle methods for the computation of three-dimensional incompressible unsteady flows. J. Comput. Phys. 109, 247273. 


\section{University Library}

\section{- M M N E R VA A gateway to Melbourne's research publications}

Minerva Access is the Institutional Repository of The University of Melbourne

Author/s:

Perry, A. E.;Marusic, I.

Title:

A wall-wake model for the turbulence structure of boundary layers. Part 1. Extension of the attached eddy hypothesis

Date:

1995

Citation:

Perry, A. E. \& Marusic, I. (1995). A wall-wake model for the turbulence structure of boundary layers. Part 1. Extension of the attached eddy hypothesis. Journal of Fluid Mechanics, 298, 361-388.

Publication Status:

Published

Persistent Link:

http://hdl.handle.net/11343/34807 